\title{
On the Finite Element Method for Singularly Perturbed Reaction-Diffusion Problems in Two and One Dimensions*
}

\author{
By A. H. Schatz and L. B. Wahlbin
}

\begin{abstract}
Second order elliptic boundary value problems which are allowed to degenerate into zero order equations are considered. The behavior of the ordinary Galerkin finite element method without special arrangements to treat singularities is studied as the problem ranges from true second order to singularly perturbed.
\end{abstract}

1. Introduction. Consider the problem of finding $u=u(x)=u\left(x_{1}, x_{2} ; \varepsilon\right)$ such that

$$
\begin{gathered}
-\varepsilon^{2} \Delta u+b(x, u ; \varepsilon)=f(x ; \varepsilon) \text { in } \Re, \\
u \in \mathscr{B},
\end{gathered}
$$

where $R$ is a bounded plane domain, $0<\varepsilon \leqslant 1$ a parameter and where $u \in \mathscr{B}$ designates some boundary conditions.

Allowing the full range of $\varepsilon$ we obtain pointwise local and global error estimates for the ordinary Galerkin finite element method with a family of quasi-uniform, unrefined meshes. These estimates are used to investigate the performance of the method.

Assumptions. We shall now more precisely describe the problems (1.1) that will be included in our analysis. For the moment we list basic minimal assumptions sufficient to derive our main results below. In applications of these, further conditions may be imposed.

For the domain $R$ we assume either

$\Re$ is a convex polygon with straight edges,

or

$$
\partial \Re \text { is of class } e^{1,1} \text {. }
$$

The boundary conditions are, for ease of exposition, restricted to Dirichlet or Neumann type, i.e.,

$$
u=F \quad \text { on } \partial \Re
$$

or

$$
\frac{\partial u}{\partial n}=F \quad \text { on } \partial \Re
$$

Received March 4, 1982.

1980 Mathematics Subject Classification. Primary 65N30, 35B25.

* Supported by the National Science Foundation. 
In the rest of this introduction we shall furthermore treat only homogeneous conditions, $F=0$. The nonhomogeneous case will be given in Section 9 .

When applying our main results we shall treat the Dirichlet problem, leaving the generally better behaved Neumann case to the reader.

For the function $b(x, u ; \varepsilon)$ it is essentially required that $\partial b / \partial u$ is positive uniformly in $u$. The nonlinear case will be given in Section 12, and for the remainder of this introduction we shall consider the linear case. Thus, (1.1.a) is replaced by

$$
L_{\varepsilon} u \equiv-\varepsilon^{2} \Delta u+a(x ; \varepsilon) u=f(x ; \varepsilon) \text { in } \Re,
$$

where $a(x ; \varepsilon)$ is measurable and, with positive constants $a_{0}, a_{1}$,

$$
0<a_{0} \leqslant a(x ; \varepsilon) \leqslant a_{1}, \quad x \in \overline{\mathcal{R}}, 0<\varepsilon \leqslant 1 .
$$

The functions $f(x ; \varepsilon)$ are assumed to be uniformly bounded in $L_{2}(\Re)$,

$$
\|f\|_{L_{2}}<C, \quad 0<\varepsilon \leqslant 1 .
$$

Numerical Method. For the numerical solution of (1.1.a $)_{\text {lin }},(1.1 . b)$, we introduce a quasi-uniform family of partitions of $\mathcal{R}$, with elements of diameter comparable to a parameter $h, 0<h \leqslant 1 / 2$, and finite element function spaces $\delta_{h}$. The detailed description of these matters is given in Section 3. We find the Galerkin approximation $u_{h} \in \mathcal{S}_{h}$ via

$$
A_{\varepsilon}\left(u_{h}, \chi\right) \equiv \varepsilon^{2}\left(\nabla u_{h}, \nabla \chi\right)+\left(a u_{h}, \chi\right)=(f, \chi), \text { for } \chi \in \delta_{h},
$$

where $(v, w)=\int_{\Re} v \cdot w$. For simplicity in the analysis it is assumed throughout this paper that the mesh domains coincide with the basic domain $R$ and that all integrals are evaluated exactly.

Main Results. We now display the two fundamental estimates of this paper. First, we have the global result that there exists a constant $C$, independent of $\varepsilon, u$ and $h$, such that, Theorem 6.1,

$$
\left\|u-u_{h}\right\|_{L_{\infty}(\Re)} \leqslant \ln (C+\varepsilon / h) \min _{\chi \in \delta_{h}}\|u-\chi\|_{L_{\infty}(\Re)} .
$$

This result is localized as follows. Let $x_{0} \in \overline{\mathcal{R}}$, let $D$ be a disc of radius $d$ around $x_{0}$ and set $\Omega_{d}=D \cap \mathcal{R}$. There exist positive constants $c_{1}, c_{2}$ and $C$, independent of $\varepsilon$, $u, h, x_{0}$ and $d$, such that if $d \geqslant c_{1} h$, Theorem 7.1,

$$
\begin{aligned}
& \left|\left(u-u_{h}\right)\left(x_{0}\right)\right| \leqslant \ln ^{1 / 2}(d / h) \\
& \quad \times\left\{\ln (C+\varepsilon / h) \min _{\chi \in \mathbb{S}_{h}}\|u-\chi\|_{L_{\infty}\left(\Omega_{d}\right)}+\frac{C}{d} e^{-c_{2} d /(\varepsilon+h)}\left\|u-u_{h}\right\|_{L_{2}\left(\Omega_{d}\right)}\right\} .
\end{aligned}
$$

The results in the nonlinear problem are quite similar, see Section 12.

Comparison With Some Other Finite Element Work. Let us comment on these estimates in the light of previous work on Galerkin finite element methods. In (1.7), when $\varepsilon \sim 1$ we recover an almost best approximation result in the maximum norm for elliptic projections derived in Schatz and Wahlbin [25]. (For certain "higher order" element spaces, the logarithmic factor can be replaced with a constant $C$; this we have not included in the present work since it would considerably lengthen it.) Taking formally $\varepsilon=0$, we recognize the almost best approximation property of the $L_{2}$ projection; see Descloux [7] or Douglas, Dupont and Wahlbin [8]. 
In the local estimate (1.8), for $\varepsilon$ of unit size we have (modulo logarithmic factors which are again there to shorten the analysis) the local estimates of [24]. When formally $\varepsilon=0$, the reader may compare with the exponential decay results for the $L_{2}$ projection given in [7] and [8].

The present unified proof is based on the techniques of Nitsche and Schatz [21] and those of [25].

Example of Local Best Approximation. We now give an example of how the local estimate (1.8) can be applied to a Dirichlet problem in our general, possibly nonsmooth, situation. Let $\Omega_{0} \subseteq \Omega_{1} \subseteq \Re$ be domains with $\operatorname{dist}\left(\Omega_{0}, \partial \Omega_{1}\right)=d>0$. By Theorem A.1(i), which is a straightforward energy estimate, and by (1.5),

$$
\left\|u-u_{h}\right\|_{L_{2}(\Re)} \leqslant C \min \left(1, h^{2} / \varepsilon^{2}\right)\|f\|_{L_{2}(\Re)} \leqslant C \min \left(1, h^{2} / \varepsilon^{2}\right) .
$$

Note next the following elementary inequalities.

$$
\begin{aligned}
& \min \left(1, h^{2} / \varepsilon^{2}\right) e^{-c d /(\varepsilon+h)} / d \\
& \leqslant \begin{cases}h^{2} / \varepsilon^{2} d & \text { for } \varepsilon \text { and } d \text { of unit size, } \\
h^{2} e^{-c d / 2 \varepsilon} / \varepsilon^{2} d \leqslant C h^{2} & \text { for } 1 / 2 \geqslant \varepsilon \geqslant h, d \geqslant \frac{8}{c} \varepsilon \ln (1 / \varepsilon), \\
e^{-c d / 2 h} / d \leqslant C h^{2} & \text { for } \varepsilon \leqslant h, d \geqslant \frac{8}{c} h \ln (1 / h) .\end{cases}
\end{aligned}
$$

Substituting the above in (1.8), we have for $d$ either of unit size or $d \geqslant$ const $\max (\varepsilon \ln (1 / \varepsilon), h \ln (1 / h))$,

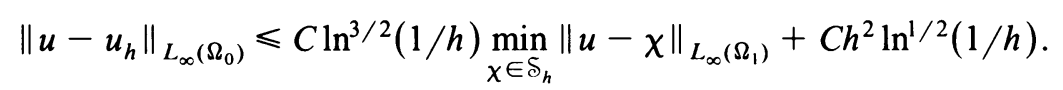

We conclude that, under our minimal smoothness assumptions (1.2), (1.4), (1.5), the Galerkin solution performs in a locally optimal fashion on subdomains, up to the order $h^{2}$ determined by the second term on the right. For $\varepsilon$ small, these subdomains can be close to regions where the solution is very rough.

In Section 10 we shall give further explicit estimates under additional smoothness assumptions.

Remark 1.1. The factor $h^{2}$ in (1.9) can be replaced by $h^{r}$ for piecewise polynomial element spaces of degree $r-1$, provided either $0<\varepsilon \leqslant c / \ln (1 / h)$ or, for a result uniformly in the full range $0<\varepsilon \leqslant 1$, if data in the problem are smoother.

Matched Asymptotic Expansions. One classical method of obtaining approximations to (1.1.a) $)_{\text {lin }}$, (1.1.b) for small $\varepsilon$ is via matched asymptotic expansions. We start by recalling salient points concerning these techniques. Comparing them with our estimates for the Galerkin method, one can determine, in terms of $h$ and $\varepsilon$, which of the two approximations is asymptotically better. We shall do this only from the point of view of global maximum norm estimates. We consider the homogeneous Dirichlet problem (in which the boundary layer is more pronounced than in a Neumann problem). Further smoothness conditions have to be imposed. Assume that

$$
\partial \mathscr{R} \text { is of class } e^{2, \alpha} \text {, some } \alpha>0 \text {, }
$$


and

$$
a(x ; \varepsilon), \quad f(x ; \varepsilon) \in \mathcal{C}^{2}, \quad \text { uniformly in } \varepsilon .
$$

Let $\partial(x)$ denote the distance from $x$ to $\partial \Re$ along the normal to $\partial R$, let $x^{\prime}$ denote the point where the normal meets $\partial \mathscr{R}$, and let $\psi(x)$ be a smooth cutoff function isolating a layer around $\partial \Re$. Set

$$
u_{\varepsilon}(x):=\frac{f(x)}{a(x)}-\frac{f\left(x^{\prime}\right)}{a\left(x^{\prime}\right)} \exp \left(-\frac{\partial(x)}{\varepsilon} \sqrt{a\left(x^{\prime}\right)}\right) \psi(x) .
$$

The functions $a$ and $f$ could be evaluated at the current $\varepsilon$, or at $\varepsilon=0$. The first term on the right is called the "regular expansion", the second term is the "boundary layer correction". We have given only the first term of each; the second term of the boundary correction is hard to compute in general. The terminology "outer" and "inner" expansions is also frequently used.

The problem of making such expansions rigorously valid as approximations to $u$ for small $\varepsilon$ has been studied, e.g., in Besjes [4], Eckhaus [9, Section 2.5.3], [10, Section 7.1.1] and Lions [14, Chapter II]. The thickness of the boundary layer can be argued to be $O(\varepsilon \ln (1 / \varepsilon))$, cf. Baranger [1]. Under the assumptions (1.10) and (1.11) one effortlessly verifies, using a "normal-tangential" coordinate system in a strip around the boundary, that $L_{\varepsilon}\left(u-u_{\varepsilon}\right)=O(\varepsilon)$. Since $u-u_{\varepsilon}=0$ on $\partial \Re$ and $\Delta\left(u-u_{\varepsilon}\right) \leqslant 0$ $(\geqslant 0)$ at an interior positive maximum (negative minimum), it follows that

$$
\left\|u-u_{\varepsilon}\right\|_{L_{\infty}(\Re)} \leqslant a_{0}^{-1} O(\varepsilon) \leqslant C \varepsilon .
$$

We shall next see that, under the smoothness assumptions (1.10) and (1.11),

$$
\left\|u-u_{h}\right\|_{L_{\infty}(\Re)} \leqslant C_{\delta} \ln (C+\varepsilon / h) h^{2} \varepsilon^{-2-\delta} \text { for } \delta>0 .
$$

By (1.7) it suffices to estimate $\|u-\chi\|_{L_{\infty}}$ for suitable $\chi$, and, for typical finite element spaces $\delta_{h}$, this quantity is bounded by $C h^{2}\|u\|_{\mathcal{C}^{2}}$. To estimate $\|u\|_{\mathcal{C}^{2}}$, one has by classical Schauder estimates, cf. e.g., Bers, John and Schechter [3, Part II, 5.6],

$$
\|u\|_{e^{2}} \leqslant C_{\alpha} \varepsilon^{-2}\left(\|f\|_{e^{\alpha}}+\|u\|_{e^{\alpha}}\right) \text {. }
$$

By [3, Part II, 5.2, Lemma 1], $\|u\|_{e^{\alpha}} \leqslant \mu\|u\|_{e^{2}}+C \mu^{-\alpha /(2-\alpha)}\|u\|_{L_{\infty}}$ whereupon choosing $\mu=\varepsilon^{2} / 2 C_{\alpha}$ and using that $\|u\|_{L_{\infty}} \leqslant a_{0}^{-1}\|f\|_{L_{\infty}}$,

$$
\|u\|_{e^{2}} \leqslant C_{\delta} \varepsilon^{-2-\delta}, \quad \delta>0
$$

which proves (1.14). The ideas above are taken from Besjes [4, Theorem 6].

Comparing (1.13) and (1.14) one sees that it is favorable to switch from the finite element solution to the matched asymptotic expansion when $\varepsilon$ goes below $O\left(h^{2 / 3}\right)$. Other combinations of $u_{h}$ and $u_{\varepsilon}$ may be used, e.g., a uniform almost $h^{r}$ accuracy of $u_{h}$ on interior domains, cf. (1.9), may be combined with the resolution of the boundary layer afforded by $u_{\varepsilon}$. From the point of view of interior accuracy the finite element solution would then be employed until $\varepsilon \sim h^{r}$.

Still other combined uses of the asymptotic expansion and the finite element method on a fixed mesh can readily be envisioned: If, in a problem with smooth data, the quantities $u_{\varepsilon}$ and $L_{\varepsilon} u_{\varepsilon}$ are easily computable, then the Galerkin method could be employed to find an approximation $\left(u-u_{\varepsilon}\right)_{h}$ to $\left(u-u_{\varepsilon}\right)$. Our main results estimate the error in this. Since, for small $\varepsilon, u-u_{\varepsilon}$ exhibits a weaker boundary layer 
than $u$ does, the deviation in the approximation $\left(u-u_{\varepsilon}\right)_{h}+u_{\varepsilon}$ for $u$ might be smaller than that in $u_{h}$. We leave it to the interested reader to pursue this idea of subtracting the singularity in more detail.

We emphasize again that the asymptotic expansion is not valid unless data in the problem are somewhat smooth.

Some Numerical Analysis Work on Reaction-Diffusion Problems. Let us now briefly comment on other work in the numerical analysis of (1.1.a $)_{\operatorname{lin}},(1.1 . b)$. This work is mostly done in one-dimensional situations and with enough smoothness present to guarantee asymptotic expansions for small $\varepsilon$. Fitting the form of the boundary layer is considered (for one-dimensional convection-diffusion equations; the ideas are analogous) in Hemker [12] and Miranker [18, Section 11]. An automatic mesh-refinement procedure is given in Reinhardt [22].

Another circle of ideas, first given for convection-diffusion equations in the well-known paper by Ilin [13], is pursued in one-dimensional cases in Shiskin and Titov [26], Miller [17] and Niijima [19], [20]. Computations are done on a mesh independent of $\varepsilon$ (as with us), say a uniform mesh, and specially designed finite difference schemes are used. The concept of convergence uniformly in $\varepsilon$ is formalized as follows.

Convergence Uniformly in $\varepsilon$. A family of approximations $u_{h}, 0<h \leqslant 1 / 2$, converges uniformly to $u$ in the norm (or seminorm) $\|\quad\|$ to order $\mu$ if, with a constant $C$ independent of $\varepsilon$ and $h$,

$$
\sup _{0<\varepsilon \leqslant 1}\left\|u-u_{h}\right\| \leqslant C h^{\mu} .
$$

The specially constructed finite difference approximations alluded to above were shown to converge uniformly in $\varepsilon$ to various orders in the meshnorm $\|v\|=\|v\|_{h}$ $=\max \{|v(x)|: x$ meshpoint $\}$. It appears hard to extend these methods to twodimensional situations since they are all based on an ability to solve the equation with constant coefficient $a(x ; \varepsilon)$ by quadrature. (A dimension-splitting procedure might be feasible.)

Considering the form of the boundary layer term in (1.12), one realizes that, in a general Dirichlet problem, the finite element method on a quasi-uniform family of meshes cannot converge uniformly in $\varepsilon$ in the global norm \|\|$_{L_{\infty}(\Re)}$. The estimate (1.9) gives an interior uniform convergence result of order almost $r$, provided $u \in \mathcal{C}^{r}\left(\Omega_{1}\right)$. For a smooth problem, the method embodied in (1.13) and (1.14), i.e., switching from the Galerkin approximation $u_{h}$ to the matched expansion $u_{\varepsilon}$ when $\varepsilon \leqslant O\left(h^{2 / 3}\right)$, gives a "method" of uniform order almost $2 / 3$ in the global maximum norm.

Pollution Absorbent Mesh. The local estimate (1.8) suggests an interpretation of the error at a point as governed by local approximability of $u$ and, for $\varepsilon$ and $h$ small, a decaying pollution effect from remote rough spots, including the boundary layer. For $\varepsilon \leqslant h$, the influence of pollution from the singularly perturbed zone extends a distance $h \ln (1 / h)$ rather than the shorter distance $\varepsilon \ln (1 / \varepsilon)$. The situation is analogous to the fairly well-known case of the centered difference scheme on a uniform mesh. If one computes with the Galerkin method for small $\varepsilon$, the fact that the distance $h \ln (1 / h)$ can be taken in terms of the local mesh size motivates the practical recipe of "sacrificing" a few more closely spaced elements around the 
boundary for the purpose of reducing the absolute size of the polluted area. (The object is not to resolve the boundary layer, which would demand a mesh changing with $\varepsilon$.) Numerical calculations in Section 11, in particular Examples 11.5 and 11.6, give some encouragement to this idea, which is trivial to implement.

$L_{2}$ Estimates. For the finite element method in a Dirichlet problem, global estimates uniformly in $\varepsilon$ can be derived in the $L_{2}$ norm. Under the assumption (1.2) on $R$, and under conditions on $a(x ; \varepsilon)$ and $f(x ; \varepsilon)$ allowing, e.g., jump discontinuities, but not imposing any boundary conditions on $f(x ; \varepsilon)$, we show in Theorem A.2 that

$$
\left\|u-u_{h}\right\|_{L_{2}(G)} \leqslant C \min \left(\sqrt{h}, h^{2} \varepsilon^{-3 / 2}\right)
$$

so that we have uniform convergence of order $\frac{1}{2}$. Other bounds are given in Theorem A.1.

Remark 1.2. Switching to $\tilde{u}_{\varepsilon}:=f(x) / a(x)$ when $\varepsilon \leqslant h$, one can prove that, under the same conditions on $a(x ; \varepsilon)$ and $f(x ; \varepsilon)$ as in Theorem A.2, the right-hand side in (1.15) can be replaced by $C \min \left(\sqrt{\varepsilon}, h^{2} \varepsilon^{-3 / 2}\right)$.

Extensions of Present Work. We comment here briefly on some simple extensions of the present investigation that we have not included for reasons of length. Two such results were already given in Remarks 1.1 and 1.2.

(i) The local estimate (1.8) is only influenced by quasi-uniformity conditions in terms of a local meshsize on $\Omega_{d}$ and the size or variation of $a(x ; \varepsilon)$ on $\Omega_{d}$. It could thus be applied to analyze certain meshrefinements and also to problems with turning points, $a\left(x_{0} ; \varepsilon\right)=0$, away from the turning point: if $a(x ; \varepsilon) \sim \alpha$ on $\Omega_{d}$, the result (1.8) holds with $\varepsilon$ replaced by $\varepsilon / \sqrt{\alpha}$. Further, we could allow $\varepsilon$ to vary over the domain.

(ii) The estimates (1.7) and (1.8) extend in a straightforward manner to many situations in which the problem $-\Delta u+v=g, g \in \mathfrak{B}$, enjoys $H^{2}$ regularity for $g$ in $L_{2}$. As an example, one has the case of $\mathcal{R}$ a rectangle with mixed boundary conditions, of Dirichlet or Neumann type on each side. Third-type boundary conditions could also be treated.

(iii) The term $-\varepsilon^{2} \Delta u$ in (1.1.a) could be replaced by

$$
-\varepsilon^{2} \nabla \cdot\left(K_{1}(x ; \varepsilon) \nabla u\right)+K_{2}(x ; \varepsilon) \cdot \nabla u
$$

with $K_{1}$ positive and $\left|K_{2}\right| \leqslant \varepsilon^{\gamma}$ with $\gamma>1$.

Outline of the Paper. Sections 2-9 are concerned with the fundamental estimates (1.7) and (1.8) in the linear case. In this, Sections 2-5 are preparatory. In Section 2 some estimates for the continuous problem are collected. The assumptions on the finite element spaces are displayed in Section 3. Simple global energy error estimates are derived in Section 4 and Section 5 is concerned with local energy estimates for the discrete problem and for the error.

In Section 6 we then prove the global best approximation result of (1.7) and, in Section 7, its localization (1.8). Modifications necessary to derive the analogous results in the one-dimensional case are given in Section 8; there are no logarithmic factors in these estimates. Nonhomogeneous boundary conditions are treated in Section 9. 
The local estimate is made explicit, for smooth Dirichlet problems, in Section 10. Numerical illustrations occupy Section 11 and the nonlinear case is given in Section 12.

In Appendix 1 the uniform global $L_{2}$ estimate (1.15) and other $L_{2}$ estimates are derived in Dirichlet problems.

Some Notation. We fix notation that shall be used throughout the paper. We employ the conventional spaces $L_{p}(\Omega), W_{p}^{k}(\Omega), H^{k}(\Omega)=W_{2}^{k}(\Omega)$ and $\bigodot^{k}(\Omega)$ and their norms; as usual, $\dot{H}^{k}(\Omega)$ denotes the closure of $\mathcal{C}_{0}^{\infty}(\Omega)$ in the $H^{k}(\Omega)$ norm. For brevity we write

$$
\|v\|_{\Omega}=\|v\|_{L_{2}(\Omega)}
$$

and, when $\Omega=\Re$, the basic domain, we sometimes leave out the region so that

$$
\|v\|_{W_{P}^{k}}=\|v\|_{W_{P}^{k}(\Re)} \text { and }\|v\|=\|v\|_{L_{2}(\Re)} .
$$

We also use the notation $|v|_{W_{p}^{k}(\Omega)}$ for the corresponding seminorms, with the same conventions as for the full norms.

As special notation we let

$$
\mathscr{H}^{1}=H^{1}(\Re) \text { or } \stackrel{H}{ }^{1}(\Re)
$$

according to whether a homogeneous Neumann or Dirichlet problem is under consideration. Correspondingly, with $S_{h}$ a "basic" finite element space and $\dot{S}_{h}$ those functions in $S_{h}$ that vanish on $\partial \Re$, we let $\mathcal{S}_{h}=S_{h}$ or $\dot{S}_{h}$ depending on the boundary condition, cf. Section 3.

The symbol $L_{\varepsilon}$ shall always designate the linear operator $L_{\varepsilon} v=-\varepsilon^{2} \Delta v+a(x ; \varepsilon) v$, and $A_{\varepsilon}(v, w)$ shall stand for the bilinear form

$$
A_{\varepsilon}(v, w)=\varepsilon^{2} \int_{\mathscr{R}} \nabla v \cdot \nabla w+\int_{\mathscr{R}} a v w .
$$

Finally, with $\Omega_{0} \subseteq \Omega_{1} \subseteq \Re$ we set

$$
\partial_{\Varangle}\left(\Omega_{0}, \Omega_{1}\right)=\operatorname{dist}\left(\partial \Omega_{0} \backslash \partial \Re, \partial \Omega_{1} \backslash \partial \Re\right) .
$$

Acknowledgement. We thank L. Bales and P. Chavez for their generous help in the numerical calculations.

2. Some Estimates for the Continuous Problem. In this section we establish some results for the problem $L_{\varepsilon} v \equiv-\varepsilon^{2} \Delta v+a(x ; \varepsilon) v=g$ in $\Re, v \in \Re$, subject to the conditions (1.2), (1.3) and (1.4). The weak form of this is to find $v \in \mathcal{H}^{1}$ such that

$$
A_{\varepsilon}(v, w) \equiv \varepsilon^{2}(\nabla v, \nabla w)+(a v, w)=(g, w) \text { for } w \in \mathcal{H}^{1},
$$

where $\mathcal{H}^{1}=\stackrel{\circ}{H}^{1}$ and $\mathcal{H}^{1}=H^{1}$ in the cases of homogeneous essential Dirichlet boundary conditions and natural Neumann conditions, respectively.

We first collect some a priori estimates.

LEMMA 2.1. There exists a constant $C$ such that, for $v$ the solution of (2.1),

$$
\begin{gathered}
\|v\| \leqslant C\|g\|, \\
\|v\|_{H^{1}} \leqslant \frac{C}{\varepsilon}\|g\|, \\
\|v\|_{H^{2}} \leqslant \frac{C}{\varepsilon^{2}}\|g\| \quad \text { for } 0<\varepsilon \leqslant 1 .
\end{gathered}
$$


Proof. We have from (2.1),

$$
\varepsilon^{2}\|\nabla v\|^{2}+a_{0}\|v\|^{2} \leqslant A_{\varepsilon}(v, v)=(g, v) \leqslant\|g\|\|v\|
$$

so that $\|v\| \leqslant a_{0}^{-1}\|g\|$ and, consequently, $\|\nabla v\| \leqslant a_{0}^{-1 / 2} \varepsilon^{-1}\|g\|$. This proves (2.2) and (2.3).

We next recall, cf. e.g. Grisvard [11, Theorems 3.2.1.2, 3.2.1.3 and 4.3.1.4] that, for the boundary conditions and types of domains under consideration, $v \in H^{2}$ (for $\left.g \in L_{2}\right)$ and

$$
\|v\|_{H^{2}} \leqslant C\|-\Delta v+v\|
$$

Hence, by use of (2.2),

$$
\|v\|_{H^{2}} \leqslant \frac{C}{\varepsilon^{2}}\left\|L_{\varepsilon} v\right\|+C\left(\frac{a_{1}}{\varepsilon^{2}}+1\right)\|v\| \leqslant \frac{C}{\varepsilon^{2}}\|g\|
$$

which completes the proof of the lemma.

We next derive a result concerning exponential decay for functions which satisfy $L_{\varepsilon} v=0$ in a subregion of $R$.

LEMMA 2.2. There exist positive constants $c$ and $C$ such that the following holds:

Let $\Omega_{0} \subseteq \Omega_{1} \subseteq \Re$ with, cf. (1.17), $d=\partial_{\Varangle}\left(\Omega_{0}, \Omega_{1}\right)>0$, and let $v \in \Re, L_{\varepsilon} v=0$ on $\Omega_{1}$. Then, for $0<\varepsilon \leqslant 1$,

$$
\begin{gathered}
\|v\|_{\Omega_{0}}+d\|\nabla v\|_{\Omega_{0}} \leqslant C e^{-c d / \varepsilon}\|v\|_{\Omega_{1}}, \\
\|\nabla v\|_{\Omega_{0}} \leqslant C e^{-c d / \varepsilon}\|\nabla v\|_{\Omega_{1}} .
\end{gathered}
$$

Proof. Let $D_{\rho}$ and $D_{\rho+\delta}$ be two concentric discs of radii $\rho$ and $\rho+\delta$, respectively, with center in $\Omega_{0}$, and let $\omega \in \mathcal{C}_{0}^{\infty}\left(D_{\rho+\delta}\right)$ be such that

$$
\omega \equiv 1 \quad \text { on } D_{\rho}, \quad\|\omega\|_{\mathcal{C}^{k}\left(D_{\rho+\delta}\right)} \leqslant C \delta^{-k}, \quad k=0,1,2,
$$

with $C$ independent of $\rho, \delta$. Set $B_{r}=\Re \cap D_{r}$ and assume that $L_{\varepsilon} v=0$ on $B_{\rho+\delta}$. We have

$$
\begin{aligned}
\varepsilon^{2}\|\omega \nabla v\|^{2}+a_{0}\|\omega v\|^{2} & \leqslant \varepsilon^{2}\left(\nabla v, \omega^{2} \nabla v\right)+\left(a v, \omega^{2} v\right) \\
& =A_{\varepsilon}\left(v, \omega^{2} v\right)-2 \varepsilon^{2}(\omega \nabla v,(\nabla \omega) v) .
\end{aligned}
$$

Since $L_{\varepsilon} v=0$ on $B_{\rho+\delta}$ and $v \in \Re$, we find that $A_{\varepsilon}\left(v, \omega^{2} v\right)=0$ so that, using (2.8),

$$
\varepsilon^{2}\|\omega \nabla v\|^{2}+a_{0}\|\omega v\|^{2} \leqslant \frac{\varepsilon^{2}}{2}\|\omega \nabla v\|^{2}+\frac{C \varepsilon^{2}}{\delta^{2}}\|v\|_{B_{\rho+\delta}}^{2}
$$

and, consequently, with $K$ independent of $\varepsilon, \rho$ and $\delta$,

$$
\begin{aligned}
\|\nabla v\|_{B_{\rho}} & \leqslant \frac{K}{\delta}\|v\|_{B_{\rho+\delta}}, \\
\|v\|_{B_{\rho}} & \leqslant \frac{K \varepsilon}{\delta}\|v\|_{B_{\rho+\delta}} .
\end{aligned}
$$

We shall now show that

$$
\|v\|_{\Omega_{0}} \leqslant C e^{-c d / \varepsilon}\|v\|_{\Omega_{1}}
$$

Assume first that

$$
d \geqslant 4 \text { Ke } \varepsilon .
$$


We obtain via iteration of (2.11) with $\delta=K e \varepsilon, \rho=\delta, 2 \delta, \ldots, N \delta$,

$$
\|v\|_{B_{\rho}} \leqslant e^{-1}\|v\|_{B_{2 \delta}} \leqslant e^{-N}\|v\|_{B_{(N+1) \delta}},
$$

where, to guarantee that $L_{\varepsilon} v=0$ on $B_{(N+1) \delta}$, we insist that

$$
(N+1) \delta \leqslant d, \text { i.e., }(\operatorname{Ke\varepsilon })(N+1) \leqslant d .
$$

Clearly, with (2.13), the largest such integer $N$ satisfies

$$
N \geqslant \frac{1}{2 K e} \cdot \frac{d}{\varepsilon} \text {. }
$$

Now cover $\Omega_{0}$ in a locally finite way with discs of radius $\delta$. Upon squaring and summing (2.14), (2.12) obtains, under the condition (2.13), with $c=(2 \mathrm{Ke})^{-1}$ and $C$ depending on the disc-covering procedure. Since (2.12) is trivial for $d<4 \operatorname{Ke\varepsilon }$, it follows in general.

To show the full inequality (2.6), one first takes (2.10) into account, setting there $\delta=d / 2$, and one then proceeds as for (2.12).

For (2.7), one uses (2.9) to obtain

$$
\begin{aligned}
\varepsilon^{2}\|\omega \nabla v\|^{2}+a_{0}\|\omega v\|^{2} & \leqslant-2 \varepsilon^{2}(\nabla \omega \cdot \nabla v, \omega v) \\
& \leqslant \frac{C \varepsilon^{4}}{\delta^{2}}\|\nabla v\|_{B_{\rho+\delta}}^{2 \cdot}+\frac{a_{0}}{2}\|\omega v\|^{2}
\end{aligned}
$$

so that

$$
\|\nabla v\|_{B_{\rho}} \leqslant \frac{C \varepsilon}{\delta}\|\nabla v\|_{B_{\rho+\delta}} .
$$

One then continues essentially as before.

This proves the lemma.

We conclude this section with the following technical local regularity result for functions satisfying $L_{\varepsilon} v=0$ in a subset.

LEMMA 2.3. There exists a constant $C$ such that the following holds:

Let $\Omega_{0} \subseteq \Omega_{1} \subseteq \Re$ with $d=\partial_{\Varangle}\left(\Omega_{0}, \Omega_{1}\right)>0$, and let $v \in \Re, L_{\varepsilon} v=0$ on $\Omega_{1}$. Then

$$
\varepsilon^{2}\|v\|_{H^{2}\left(\Omega_{0}\right)} \leqslant C\left(\frac{\varepsilon^{2}}{d}\|\nabla v\|_{\Omega_{1}}+\|v\|_{\Omega_{1}}\right), \quad 0<\varepsilon \leqslant 1 .
$$

Proof. It is clearly enough to show (2.15) with the left-hand side replaced by the seminorm $|v|_{H^{2}\left(\Omega_{0}\right)}$. Reasoning as in the proof of Lemma 2.2 and using notation therefrom, it suffices to prove

$$
\varepsilon^{2}|v|_{H^{2}\left(B_{\delta}\right)} \leqslant C\left(\frac{\varepsilon^{2}}{\delta}\|\nabla v\|_{B_{3 \delta}}+\|v\|_{B_{3 \delta}}\right), \quad \text { with } \delta=d / 3 .
$$

Let $\omega$ be as in (2.8), with $\rho=\delta$, and let further $M$ denote the mean-value of $v$ over $B_{2 \delta}$, i.e.,

$$
M=\left(\operatorname{meas} B_{2 \delta}\right)^{-1} \int_{B_{2 \delta}} v
$$

It is well known that

$$
\|v-M\|_{B_{2 \delta}} \leqslant C \delta\|\nabla v\|_{B_{3 \delta}},
$$

where $C$ does not depend on $\delta$. 
We now first treat the case of Dirichlet boundary conditions. Assume first that supp $\omega$ does not intersect $\partial \Re$. Then by (2.5),

$$
\begin{aligned}
\varepsilon^{2}|v|_{H^{2}\left(B_{\delta}\right)} & =\varepsilon^{2}|v-M|_{H^{2}\left(B_{\delta}\right)} \leqslant \varepsilon^{2}|\omega(v-M)|_{H^{2}} \\
& \leqslant C\left\|\varepsilon^{2} \Delta(\omega(v-M))+\varepsilon^{2} \omega(v-M)\right\| \\
& \leqslant C \varepsilon^{2}\|(\Delta \omega+\omega)(v-M)\|+C \varepsilon^{2}\|\nabla \omega \cdot \nabla v\|+C\left\|\omega \varepsilon^{2} \Delta v\right\| \\
& \leqslant \frac{C \varepsilon^{2}}{\delta^{2}}\|v-M\|_{B_{2 \delta}}+\frac{C \varepsilon^{2}}{\delta}\|\nabla v\|_{B_{2 \delta}}+C\|\omega v\|,
\end{aligned}
$$

where, in the last step, we also used that $L_{\varepsilon} v=0$ on the support of $\omega$. Taking (2.17) into account, (2.16) obtains.

In case supp $\omega$ intersects $\partial \mathscr{R}$, take $M=0$ in (2.18) and estimate the term $\varepsilon^{2}\|v\|_{B_{2 \delta}} / \delta^{2}$ via Poincaré's inequality as $C \varepsilon^{2}\|\nabla v\|_{B_{3 \delta}} / \delta$.

For the Neumann problem we separate, for technical reasons, the cases (1.2.a) and (1.2.b). For a smooth boundary we use the well-known a priori estimate, cf. e.g. [11, Corr. 2.2.2.6],

$$
\|w\|_{H^{2}} \leqslant C\|-\Delta w+w\|+C\left\|\frac{\partial w}{\partial n}\right\|_{H^{1 / 2}(\partial \Re)} .
$$

The work then proceeds as in (2.18), except that an additional term

$$
\varepsilon^{2}\left\|\frac{\partial}{\partial n}(\omega(v-M))\right\|_{H^{1 / 2}(\partial \mathscr{R})}=\varepsilon^{2}\left\|\frac{\partial \omega}{\partial n}(v-M)\right\|_{H^{1 / 2}(\partial \mathscr{R})}
$$

has to be included. Since the $H^{1 / 2}(\partial \Re)$ norm can be bounded by the $H^{1}$ norm, the proof is easily completed.

For the case of a Neumann problem on a convex straightedged polygonal domain, we have, for $w$ with $\partial w / \partial n=0$ on $\partial \Re$, cf. (2.5),

$$
\|w\|_{H^{2}} \leqslant C\|-\Delta w+w\| \text {. }
$$

We may apply this to $w=\omega(v-M)$ provided (i), (ii)(a) or (ii)(b) below hold:

(i) $\operatorname{supp} \omega \subset \subset \Re$,

(ii) $\omega$ is a radially symmetric function centered on $\partial \Re$ in such a way that

(a) supp $\omega$ avoids any vertex, or,

(b) $\omega$ is centered at a vertex and supp $\omega$ avoids any other vertex.

The domain $B_{\delta}$ can be covered with a bounded number of sets where $\omega \equiv 1$ with $\omega$ of the types described. Proceeding as in (2.18), we infer that (2.16) holds also in this case.

This completes the proof of the lemma.

3. The Approximating Spaces. We shall briskly list our hypotheses for the finite element spaces. The assumptions A.1-A.5 below are standard and well known for quasi-uniform partitions and corresponding spaces occurring in practice, cf. e.g. Ciarlet [6]. The last assumption, A.6, is perhaps less standard.

Let $0<h \leqslant 1 / 2$ be a parameter and let $\bar{R}=\cup_{i=1}^{I(h)} \bar{\tau}_{i}^{h}$ be partitions of $\bar{R}$ into finite elements $\tau_{i}^{h}$. We are, for technical simplicity, assuming exact subdivisions of $\bar{\Re}$. For curved polynomial boundaries we have isoparametric modifications in mind; for more general boundaries we refer to Zlamal [29]. We insist that the family of 
meshes is quasi-uniform, i.e., that there exist positive constants $c_{0}$ and $C_{0}$, independent of $h$ and $i$, such that, with $\rho_{i}^{h}$ denoting the diameter of the largest inscribed disc of $\tau_{i}^{h}, c_{0} h \leqslant \rho_{i}^{h} \leqslant \operatorname{diameter}\left(\tau_{i}^{h}\right) \leqslant C_{0} h$. This condition is implicitly contained in the assumptions below.

A.1. There exists a constant $C$ such that, for $v \in W_{1}^{1}\left(\tau_{i}^{h}\right), 0<h \leqslant 1 / 2, i=$ $1, \ldots, I(h)$,

$$
\int_{\partial \tau_{i}^{h}}|v| \leqslant C\left\{h^{-1}\|v\|_{L_{1}\left(\tau_{i}^{h}\right)}+|v|_{W_{1}^{\prime}\left(\tau_{i}^{h}\right)}\right\}
$$

This condition is easily verified for quasi-uniform meshes of the usual kinds.

Let $S_{h}$ be finite-dimensional subspaces of $W_{\infty}^{1}(\Re)$ such that, for $\chi$ in $S_{h}$, $\left.\chi\right|_{\tau_{i}^{h}} \in \mathcal{C}^{2}\left(\bar{\tau}_{i}^{h}\right)$. Corresponding to our convention (1.16), we introduce $\dot{S}_{h}=\left\{x \in S_{h}\right.$ : $\chi=0$ on $\partial \Re\}$ and set

$$
\varsigma_{h}=\stackrel{\circ}{S}_{h} \text { or } S_{h}
$$

according to whether homogeneous Dirichlet or Neumann conditions are under consideration.

A.2. (Inverse property.) There exists a constant $C$ such that, for $\chi \in S_{h}, 0<h \leqslant$ $1 / 2, i=1, \ldots, I(h)$,

$$
\|\chi\|_{W_{p}^{l}\left(\tau_{i}^{h}\right)} \leqslant C h^{m-l-2(1 / q-1 / p)}\|\chi\|_{W_{q}^{m}\left(\tau_{i}^{h}\right)}
$$

for $0 \leqslant m \leqslant l \leqslant 2,1 \leqslant q \leqslant p \leqslant \infty$.

We next consider approximation properties of the finite element spaces.

A.3. (Local approximation.) There exist constants $c$ and $C$ such that the following holds: Given a function $v$ (with $v=0$ on $\partial \mathcal{R}$ in the Dirichlet case), there exists $\chi \in \mathcal{S}_{h}$ such that for $0<h \leqslant 1 / 2, i=1, \ldots, I(h)$,

$$
\|v-\chi\|_{L_{2}\left(\tau_{i}^{h}\right)}+h|v-\chi|_{H^{1}\left(\tau_{i}^{h}\right)} \leqslant C h^{2}\|v\|_{H^{2}\left(\tilde{\tau}_{i}^{h}\right)},
$$

where

$$
\tilde{\tau}_{i}^{h}=\bigcup \tau_{j}^{h}, \quad \operatorname{dist}\left(\tau_{i}^{h}, \tau_{j}^{h}\right) \leqslant c h .
$$

We introduce the piecewise seminorm

$$
|v|_{W_{p}^{k, h}}=\left(\sum_{i}|v|_{W_{p}^{k}\left(\tau_{i}^{h}\right)}^{p}\right)^{1 / p}, \quad 1 \leqslant p<\infty
$$

with the usual modification for $p=\infty$.

A.4. (Global approximation.) There exists a constant $C$ such that, given a function $v$ (with $v=0$ on $\partial \Re$ in the Dirichlet case), there exists $\chi \in \mathcal{S}_{h}$ such that, for $0<h \leqslant 1 / 2$,

$$
\|v-\chi\|_{L_{1}}+h|v-\chi|_{W_{1}^{1}}+h^{2}|v-\chi|_{W_{1}^{2, h}} \leqslant C h^{2}\|v\|_{W_{1}^{2}} .
$$

Note that a similar global approximation result in $L_{2}$ and $H^{1}$ follows from A.3.

Given a domain $\Omega \leqslant \mathscr{R}$, set $\mathcal{S}_{h}(\Omega)=\mathcal{S}_{h} \mid \Omega$ and, cf. (1.17),

$$
\delta_{h}^{\Varangle}(\Omega)=\left\{\chi \in \delta_{h}: \partial_{\Varangle}(\operatorname{supp} \chi, \Omega)>0\right\},
$$

and also

$$
e_{\Varangle}^{\infty}(\bar{\Omega})=\left\{v \in e^{\infty}(\bar{\Omega}): \partial_{\Varangle}(\operatorname{supp} v, \Omega)>0\right\} .
$$


A.5. (Superapproximation.) There exist constants $c$ and $C$ and an integer $L$ such that the following holds for $0<h \leqslant 1 / 2$ :

Let $\Omega_{0} \leqslant \Omega_{1} \leqslant \Re$ with $d \geqslant c h$, where $d=\partial_{\Varangle}\left(\Omega_{0}, \Omega_{1}\right)$. Let $\omega \in \bigodot_{\Varangle}^{\infty}\left(\bar{\Omega}_{0}\right)$ with

$$
\|\omega\|_{W_{\infty}^{\prime}\left(\Omega_{0}\right)} \leqslant \Lambda d^{-l}, \quad l=0, \ldots, L .
$$

Then, for any $\chi \in \mathcal{S}_{h}$, there exists $\psi \in \mathcal{S}_{h}^{\ddagger}\left(\Omega_{1}\right)$ such that

$$
\left\|\omega^{2} \chi-\psi\right\|_{\Omega_{1}}+h\left\|\omega^{2} \chi-\psi\right\|_{H^{1}\left(\Omega_{1}\right)} \leqslant C \Lambda h^{2}\left\{d^{-2}\|\chi\|_{L_{2}\left(\Omega_{1}\right)}+d^{-1}|\chi|_{H^{1}\left(\Omega_{1}\right)}\right\} .
$$

Our last assumption is the following Sobolev type inequality on $S_{h}$.

A.6. There exist constants $c$ and $C$ such that the following holds for $0<h \leqslant 1 / 2$ :

Let $\Omega_{0} \leqslant \Omega_{1} \leqslant \Re$ with $d \geqslant c h$, where $d=\partial_{\Varangle}\left(\Omega_{0}, \Omega_{1}\right)$. Then, for any $\chi \in S_{h}$,

$$
\|\chi\|_{L_{\infty}\left(\Omega_{0}\right)} \leqslant C(\ln (d / h))^{1 / 2}\left\{\|\chi\|_{H^{1}\left(\Omega_{1}\right)}+d^{-1}\|\chi\|_{L_{2}\left(\Omega_{1}\right)}\right\} \text {. }
$$

A proof of this, for specific $S_{h}$ and $\Omega_{0}=\Omega_{1}=\Re$ (in which case $d \sim 1$ can be taken), was given in Wendland [28, Theorem 8.3.3], cf. also Schatz-Thomée-Wahlbin [23, Lemma 1.1]. These proofs are easily extended to most practical cases and they are also simple to localize by use of local extension operators and scaling arguments. Note also that, when $d \sim h$, A.6 is a consequence of A.2.

4. Global Energy Error Estimates. For $v \in \mathcal{H}$, define $P_{h}^{\varepsilon} v \in \mathcal{S}_{h}$ by

$$
A_{\varepsilon}\left(v-P_{h}^{\varepsilon} v, \chi\right)=0 \text { for } \chi \in \mathcal{\delta}_{h},
$$

cf. the conventions (1.16), (3.1). We shall show some straightforward results; more complete $L_{2}$ estimates are given in the Appendix.

Lemma 4.1. There exists a constant $C$ such that, for $0<\varepsilon \leqslant 1,0<h \leqslant \frac{1}{2}$,

$$
\begin{aligned}
&\left\|\nabla\left(v-P_{h}^{\varepsilon} v\right)\right\| \leqslant\left\{\begin{array}{l}
C\|v\|_{H^{1}}, \\
C h\|v\|_{H^{2}},
\end{array}\right. \\
&\left\|v-P_{h}^{\varepsilon} v\right\| \leqslant\left\{\begin{array}{l}
C h\|v\|_{H^{1}}, \\
C h^{2}\|v\|_{H^{2}} .
\end{array}\right.
\end{aligned}
$$

Proof. Set $e=v-P_{h}^{\varepsilon} v$. Using (4.1) we find, for any $\chi \in \mathcal{S}_{h}$,

$$
\varepsilon^{2}\|\nabla e\|^{2}+a_{0}\|e\|^{2} \leqslant A_{\varepsilon}(e, e)=A_{\varepsilon}(e, v-\chi)
$$

and hence,

$$
\varepsilon^{2}\|\nabla e\|^{2}+a_{0}\|e\|^{2} \leqslant C\left\{\varepsilon^{2}\|\nabla(v-\chi)\|^{2}+\|v-\chi\|^{2}\right\} .
$$

We first treat the case $\varepsilon \geqslant c h$, with $c$ to be determined. Taking $\chi=P_{h}^{l} v$ in (4.4) we have, as is well known, $\|\nabla(v-\chi)\| \leqslant C\|v\|_{H^{\prime}}$ and $\|v-\chi\| \leqslant C h\|v\|_{H^{\prime}}$ so that

$$
\|\nabla e\|^{2} \leqslant C\left\{\|v\|_{H^{1}}^{2}+\frac{h^{2}}{\varepsilon^{2}}\|v\|_{H^{1}}^{2}\right\} \leqslant C\|v\|_{H^{1}}^{2} .
$$

The first estimate in (4.2) follows. Using, in a global fashion, the approximation $\chi$ from A.3 we also obtain the second inequality of (4.2).

We continue with a duality argument. Let $w$ be the solution of

$$
L_{\varepsilon} w=e, \quad w \in \Re .
$$


Then, for any $\chi \in \mathcal{S}_{h}$,

$$
\|e\|^{2}=A_{\varepsilon}(w-\chi, e)
$$

so that, with $\chi$ as in A.3, utilizing (2.4),

$$
\|e\|^{2} \leqslant C\left\{\varepsilon^{2}\|\nabla e\| h\|w\|_{H^{2}}+C\|e\| h^{2}\|w\|_{H^{2}}\right\} \leqslant C\left\{h\|\nabla e\|\|e\|+\frac{h^{2}}{\varepsilon^{2}}\|e\|^{2}\right\} .
$$

Hence, for $\varepsilon \geqslant c h$ with $c=2 C,\|e\| \leqslant C h\|\nabla e\|$, from which (4.3) follows via (4.2).

It remains to consider the case $\varepsilon \leqslant c h$. By (4.4) with $\chi=P_{h}^{1} v$ we have also

$$
\|e\|^{2} \leqslant C\left(\varepsilon^{2}+h^{2}\right)\|v\|_{H^{1}}^{2} \leqslant C h^{2}\|v\|_{H^{1}}^{2},
$$

which establishes the first part of (4.3). The second part is deduced by using $\chi$ from A.3.

To show (4.2) we use the inverse property A.2. For $\chi \in \mathcal{S}_{h}$,

$$
\begin{aligned}
\|\nabla e\| & \leqslant\|\nabla(v-\chi)\|+C h^{-1}\left\|P_{h}^{\varepsilon} v-\chi\right\| \\
& \leqslant\|\nabla(v-\chi)\|+C h^{-1}\|v-\chi\|+C h^{-1}\|e\| .
\end{aligned}
$$

With suitable choices of $\chi$, as before, and taking (4.3) into account we verify (4.2).

This completes the proof of the lemma.

5. A Local Energy Estimate for the Discrete Problem and a Local Energy Error Estimate. We shall first prove a discrete analogue of Lemma 2.2.

LEMma 5.1. There exist positive constants $c_{1}, c_{2}$ and $C$ such that the following holds for $0<\varepsilon \leqslant 1,0<h \leqslant \frac{1}{2}$ :

Let $\Omega_{0} \subseteq \Omega_{1} \subseteq \Re$ with $d \geqslant c_{1} h$, where $d=\partial_{\Varangle}\left(\Omega_{0}, \Omega_{1}\right)$. If $v_{h} \in \mathcal{S}_{h}\left(\Omega_{1}\right)$ is such that

$$
A_{\varepsilon}\left(v_{h}, \chi\right)=0 \text { for } \chi \in \mathcal{S}_{h}^{\ddagger}\left(\Omega_{1}\right)(c f .(3.3) \text { for notation }) \text {, }
$$

then

$$
\left\|v_{h}\right\|_{\Omega_{0}}+d\left\|\nabla v_{h}\right\|_{\Omega_{0}} \leqslant C e^{-c_{2} d /(\varepsilon+h)}\left\|v_{h}\right\|_{\Omega_{1}} .
$$

Comparing to Lemma 2.2, we have here the restriction $d \geqslant c_{1} h$. Further, when $\varepsilon \ll h$, the decay exponent is not $d / \varepsilon$ but merely $d / h$.

Using this lemma, we shall then establish the following local error estimate.

LEMMA 5.2. There exist positive constants $c_{1}, c_{2}$ and $C$ such that the following holds for $0<\varepsilon \leqslant 1,0<h \leqslant \frac{1}{2}$ :

Let $\Omega_{0} \subseteq \Omega_{1} \subseteq \Re$ with $d \geqslant c_{1} h$, where $d=\partial_{\Varangle}\left(\Omega_{0}, \Omega_{1}\right)$. Let $v \in \mathcal{H}$ and $v_{h} \in \mathcal{S}_{h}\left(\Omega_{1}\right)$ be such that

$$
A_{\varepsilon}\left(v-v_{h}, \chi\right)=0 \text { for } \chi \in \mathcal{S}_{h}^{\ddagger}\left(\Omega_{1}\right) \text {. }
$$

Then

$$
\begin{aligned}
\left\|v-v_{h}\right\|_{H^{\prime}\left(\Omega_{0}\right)} \leqslant & C \min _{\chi \in \mathcal{S}_{h}\left(\Omega_{1}\right)}\left(\|\nabla(v-\chi)\|_{\Omega_{1}}+\frac{1}{d}\|v-\chi\|_{\Omega_{1}}\right) \\
& +\frac{C}{d} e^{-c_{2} d /(\varepsilon+h)}\left\|v-v_{h}\right\|_{\Omega_{1}} .
\end{aligned}
$$

In particular, the result holds for $v_{h}=P_{h}^{\varepsilon} v, c f .(4.1)$. 
Proof of Lemma 5.1. We follow the proof of Lemma 2.2.

Let $D_{\rho}$ and $D_{\rho+\delta}$ be two concentric discs of radii $\rho$ and $\rho+\delta$, respectively, with center in $\Omega_{0}$, and let $\omega \in \mathcal{C}_{0}^{\infty}\left(D_{\rho+\delta}\right)$ be such that

$$
\omega \equiv 1 \quad \text { on } D_{\rho}, \quad\|\omega\|_{\mathcal{C}^{\prime}\left(D_{\rho+\delta}\right)} \leqslant C \delta^{-l}, \quad l=0, \ldots, L .
$$

Set $B_{r}=\Re \cap D_{r}$ and assume that

$$
A_{\varepsilon}\left(v_{h}, \chi\right)=0 \text { for } \chi \in \mathcal{S}_{h}^{\Varangle}\left(B_{\rho+\delta}\right),
$$

and furthermore that $\delta \geqslant \tilde{c} h$. The dependence of $\tilde{c}$ on previously displayed constants can be traced in the proof below, but we leave this to the reader.

Using (5.6), we have by a simple calculation,

$$
\begin{aligned}
\varepsilon^{2}\left\|\omega \nabla v_{h}\right\|^{2} & +a_{0}\left\|\omega v_{h}\right\|^{2} \leqslant \varepsilon^{2}\left(\nabla v_{h}, \omega^{2} \nabla v_{h}\right)+\left(a v_{h}, \omega^{2} v_{h}\right) \\
& =A_{\varepsilon}\left(v_{h}, \omega^{2} v_{h}\right)-\varepsilon^{2}\left(\omega \nabla v_{h}, 2(\nabla \omega) v_{h}\right) \\
& =A_{\varepsilon}\left(v_{h}, \omega^{2} v_{h}-\chi\right)-\varepsilon^{2}\left(\omega \nabla v_{h}, 2(\nabla \omega) v_{h}\right) \text { for } \chi \in \mathcal{S}_{h}^{\Varangle}\left(B_{\rho+\delta}\right) .
\end{aligned}
$$

By the superapproximation hypothesis A.5 we thus find,

$$
\begin{aligned}
\varepsilon^{2}\left\|\omega \nabla v_{h}\right\|^{2}+a_{0}\left\|\omega v_{h}\right\|^{2} \leqslant & C \varepsilon^{2}\left\|\nabla v_{h}\right\|_{B_{\rho+\delta}}\left(\frac{h}{\delta}\left\|\nabla v_{h}\right\|_{B_{\rho+\delta}}+\frac{h}{\delta^{2}}\left\|v_{h}\right\|_{B_{\rho+\delta}}\right) \\
& +C\left\|v_{h}\right\|_{B_{\rho+\delta}}\left(\frac{h^{2}}{\delta}\left\|\nabla v_{h}\right\|_{B_{\rho+\delta}}+\frac{h^{2}}{\delta^{2}}\left\|v_{h}\right\|_{B_{\rho+\delta}}\right) \\
& +C \frac{\varepsilon^{2}}{\delta}\left\|\omega \nabla v_{h}\right\|\left\|v_{h}\right\|_{B_{\rho+\delta}}
\end{aligned}
$$

Note now that, since $\delta \geqslant \tilde{c h}$,

$$
\frac{\varepsilon^{2} h}{\delta^{2}}\left\|\nabla v_{h}\right\|_{B_{\rho+\delta}}\left\|v_{h}\right\|_{B_{\rho+\delta}} \leqslant \frac{C \varepsilon^{2} h}{\delta}\left\|\nabla v_{h}\right\|_{B_{\rho+\delta}}^{2}+\frac{C \varepsilon^{2}}{\delta^{2}}\left\|v_{h}\right\|_{B_{\rho+\delta}}^{2} .
$$

Further, by use of the inverse hypothesis A.2, for $\delta \geqslant \tilde{c} h, \tilde{c}$ large enough,

$$
\frac{h^{2}}{\delta}\left\|v_{h}\right\|_{B_{\rho+\delta}}\left\|\nabla v_{h}\right\|_{B_{\rho+\delta}} \leqslant \frac{C h}{\delta}\left\|v_{h}\right\|_{B_{\rho+2 \delta}}^{2} .
$$

Also,

$$
\frac{C \varepsilon^{2}}{\delta}\left\|\omega \nabla v_{h}\right\|\left\|v_{h}\right\|_{B_{\rho+\delta}} \leqslant \frac{\varepsilon^{2}}{2}\left\|\omega \nabla v_{h}\right\|^{2}+\frac{C \varepsilon^{2}}{\delta^{2}}\left\|v_{h}\right\|_{B_{\rho+\delta}}^{2} .
$$

Reporting (5.8)-(5.10) into (5.7),

(5.11) $\frac{1}{2} \varepsilon^{2}\left\|\nabla v_{h}\right\|_{B_{\rho}}^{2}+a_{0}\left\|v_{h}\right\|_{B_{\rho}}^{2} \leqslant \frac{C \varepsilon^{2} h}{\delta}\left\|\nabla v_{h}\right\|_{B_{\rho+\delta}}^{2}+C\left(\frac{\varepsilon^{2}}{\delta^{2}}+\frac{h}{\delta}\right)\left\|v_{h}\right\|_{B_{\rho+2 \delta}}^{2}$.

Iterating the gradient term once more and using inverse properties,

$$
\begin{aligned}
\frac{\varepsilon^{2} h}{\delta}\left\|\nabla v_{h}\right\|_{B_{\rho+\delta}}^{2} & \leqslant \frac{h}{\delta}\left(C \frac{\varepsilon^{2} h}{\delta}\left\|\nabla v_{h}\right\|_{B_{\rho+2 \delta}}^{2}+C\left(\frac{\varepsilon^{2}}{\delta^{2}}+\frac{h}{\delta}\right)\left\|v_{h}\right\|_{B_{\rho+3} \delta}^{2}\right) \\
& \leqslant C\left(\frac{\delta^{2}+h^{2}}{\delta^{2}}\right)\left\|v_{h}\right\|_{B_{\rho+3 \delta}}^{2} .
\end{aligned}
$$


Similarly, iterating part of the $L_{2}$ term in (5.11), using the above,

$$
\frac{h}{\delta}\left\|v_{h}\right\|_{B_{\rho+2 \delta}}^{2} \leqslant \frac{C h}{\delta}\left(\frac{\varepsilon^{2}}{\delta^{2}}+\frac{h}{\delta}\right)\left\|v_{h}\right\|_{B_{\rho+4 \delta}}^{2} \leqslant C\left(\frac{\varepsilon^{2}+h^{2}}{\delta^{2}}\right)\left\|v_{h}\right\|_{B_{\rho+4 \delta}}^{2} .
$$

Inserting these two inequalities in (5.11) and changing notation, we thus have

$$
\frac{1}{2} \varepsilon^{2}\left\|\nabla v_{h}\right\|_{B_{\rho}}^{2}+a_{0}\left\|v_{h}\right\|_{B_{\rho}}^{2} \leqslant C\left(\frac{\varepsilon^{2}+h^{2}}{\delta^{2}}\right)\left\|v_{h}\right\|_{B_{\rho+\delta}}^{2} .
$$

Consequently, with $K$ independent of $\varepsilon, h, \rho$ and $\delta$,

$$
\left\|\nabla v_{h}\right\|_{B_{\rho}} \leqslant \frac{K}{\delta}\left\|v_{h}\right\|_{B_{\rho+\delta}}, \quad\left\|v_{h}\right\|_{B_{\rho}} \leqslant K \frac{\sqrt{\varepsilon^{2}+h^{2}}}{\delta}\left\|v_{h}\right\|_{B_{\rho+\delta}} ;
$$

in the case of $\varepsilon \leqslant h$, the first inequality has to be separately established. This is easy using first the inverse hypothesis A.2 and then the second inequality above.

The proof is now concluded as the proof of Lemma 2.2, cf. (2.10), (2.11) et seq. We leave the details.

Proof of Lemma 5.2. Again it suffices to verify the result with $\Omega_{0}$ and $\Omega_{1}$ replaced by $B_{\rho}$ and $B_{\rho+2 \delta}$ where $\delta \geqslant \tilde{c} h$, cf. the proof of the previous lemma. Let $\omega \in$ $\bigodot_{0}^{\infty}\left(D_{\rho+2 \delta}\right)$,

$$
\omega \equiv 1 \quad \text { on } D_{\rho+\delta}, \quad\|\omega\|_{\mathcal{C}^{\prime}\left(D_{\rho+2 \delta}\right)} \leqslant C \delta^{-l}, \quad l=0,1 .
$$

With $\tilde{v}=\omega v, \tilde{v}_{h}=P_{h}^{\varepsilon}(\omega v)$,

$$
\left\|v-v_{h}\right\|_{H^{\prime}\left(B_{\rho}\right)} \leqslant\left\|\tilde{v}-\tilde{v}_{h}\right\|_{H^{1}}+\left\|\tilde{v}_{h}-v_{h}\right\|_{H^{1}\left(B_{\rho}\right)} .
$$

Here, by Lemma 5.1, since $A_{\varepsilon}\left(\tilde{v}_{h}-v_{h}, \chi\right)=0$ for $\chi \in \mathcal{S}_{h}^{\Varangle}\left(B_{\rho+\delta}\right)$,

$$
\begin{aligned}
\left\|\tilde{v}_{h}-v_{h}\right\|_{H^{\prime}\left(B_{\rho}\right)} & \leqslant \frac{C}{\delta} e^{-c \delta /(\varepsilon+h)}\left\|\tilde{v}_{h}-v_{h}\right\|_{B_{\rho+\delta}} \\
& \leqslant \frac{C}{\delta} e^{-c \delta /(\varepsilon+h)}\left(\left\|\tilde{v}-\tilde{v}_{h}\right\|_{B_{\rho+\delta}}+\left\|v-v_{h}\right\|_{B_{\rho+\delta}}\right) .
\end{aligned}
$$

It remains therefore to estimate the quantity

$$
\left\|\nabla\left(\tilde{v}-\tilde{v}_{h}\right)\right\|+\frac{1}{\delta}\left\|\tilde{v}-\tilde{v}_{h}\right\| .
$$

From Lemma 4.1, using that $\delta \geqslant \tilde{c} h$, we have the bound

$$
C\|\tilde{v}\|_{H^{1}}+\frac{C h}{\delta}\|\tilde{v}\|_{H^{1}} \leqslant C\|\tilde{v}\|_{H^{1}} \leqslant C\|\nabla v\|_{B_{\rho+2 \delta}}+\frac{C}{\delta}\|v\|_{B_{\rho+2 \delta}} .
$$

Combining this with (5.13) and (5.12) completes the proof, upon writing $v-v_{h}=$ $(v-\chi)-\left(v_{h}-\chi\right)$ for $\chi \in \mathcal{S}_{h}\left(\Omega_{1}\right)$.

6. Global Almost Best Approximation in the Maximum Norm. We shall prove the following result.

Theorem 6.1. Assume that (1.2), (1.4) and A.1-A.6 of Section 3 hold. Let $u$ be a continuous function, with $u=0$ on $\partial \Re$ in the Dirichlet case, and let $u_{h} \in \mathcal{S}_{h}$ be such that

$$
A_{\varepsilon}\left(u-u_{h}, \chi\right)=0 \quad \text { for } \chi \in \mathcal{S}_{h}
$$


(i.e., $u_{h}=P_{h}^{\varepsilon} u$ ). There exists a constant $C$, independent of $\varepsilon, h, u$ and $u_{h}$ such that, for $0<\varepsilon \leqslant 1,0<h \leqslant \frac{1}{2}$,

$$
\left\|u-u_{h}\right\|_{L_{\infty}(\Re)} \leqslant \ln \left(C+\frac{\varepsilon}{h}\right) \min _{\chi \in \delta_{h}}\|u-\chi\|_{L_{\infty}(\Re)} .
$$

Note that, by Green's theorem,

$$
\begin{aligned}
A_{\varepsilon}\left(u-u_{h}, \chi\right)= & -\varepsilon^{2} \sum_{i=1}^{I(h)}\left\{\int_{\tau_{i}^{h}}\left(u-u_{h}\right) \Delta \chi+\oint_{\partial \tau_{i}^{h}}\left(u-u_{h}\right) \frac{\partial \chi}{\partial n}\right\} \\
& +\left(a\left(u-u_{h}\right), \chi\right),
\end{aligned}
$$

so that (6.1) makes sense for $u$ continuous.

The rest of this section will be devoted to the proof of this theorem. By a density argument we may assume that $u$ is continuously differentiable.

Let $x_{0}$ be an arbitrary point in $R$, and let $\tau_{0}$ denote an element such that $x_{0} \in \bar{\tau}_{0}$. By use of the inverse property A.2 we have,

$$
\begin{aligned}
\left|\left(u-u_{h}\right)\left(x_{0}\right)\right| & \leqslant\left|u\left(x_{0}\right)\right|+\left|u_{h}\left(x_{0}\right)\right| \leqslant\left|u\left(x_{0}\right)\right|+C h^{-1}\left\|u_{h}\right\|_{\tau_{0}} \\
& \leqslant C\|u\|_{L_{\infty}}+C h^{-1}\left\|u-u_{h}\right\|_{\tau_{0}} .
\end{aligned}
$$

Here,

$$
h^{-1}\left\|u-u_{h}\right\|_{\tau_{0}}=h^{-1} \sup _{\substack{\phi \in \mathcal{C}_{0}^{\infty}\left(\tau_{0}\right) \\\|\phi\|=1}}\left(u-u_{h}, \phi\right) .
$$

For each such fixed $\phi$, let $v$ solve

$$
L_{\varepsilon} v=\phi \quad \text { in } R, v \in \mathscr{B},
$$

and let $v_{h}$ denote $P_{h}^{\varepsilon} v$. The notation for $\phi, v$ and $v_{h}$ will be fixed for the rest of the proof.

We next derive two simple preliminary results concerning $v$ and $v_{h}$.

LEMMA 6.1. There exists a constant $C$, independent of $\varepsilon, h$ and $\phi$, such that

$$
\begin{gathered}
\|v\|+\left\|v_{h}\right\| \leqslant\left\{\begin{array}{l}
C, \\
C h / \varepsilon,
\end{array}\right. \\
\|\nabla v\|+\left\|\nabla v_{h}\right\| \leqslant \frac{h}{\varepsilon^{2}} \ln ^{1 / 2}\left(C+\frac{\varepsilon}{h}\right) .
\end{gathered}
$$

Proof. Since

and a similar inequality holds for $v_{h}$, the first bound in (6.7) is clear.

For the second part of (6.7), we first consider $v$. We have $\|v\|=(v, v /\|v\|)$ and letting $L_{\varepsilon} w=v /\|v\|, w \in \mathscr{B}$, hence,

$$
\|v\|=A_{\varepsilon}(v, w)=(\phi, w) .
$$

Using Sobolev's inequality in the form $\|w\|_{L_{\infty}} \leqslant C\|w\|_{H^{2}}^{1 / 2}\|w\|^{1 / 2}$ and also that $\|\phi\|_{L_{1}} \leqslant C h\|\phi\|=C h$,

$$
(\phi, w) \leqslant C h\|w\|_{H^{2}}^{1 / 2}\|w\|^{1 / 2}
$$


which by Lemma 2.1 is bounded by $C h / \varepsilon$. This shows that $\|v\| \leqslant C h / \varepsilon$, and, since by Lemmas 4.1 and $2.1\left\|v-v_{h}\right\| \leqslant C h\|v\|_{H^{1}} \leqslant C h / \varepsilon$, we obtain (6.7).

For (6.8), we start with $v_{h}$, using the analogue of (6.9). With $\Omega^{\prime}$ the intersection of $\Re$ with a disc of radius $\max (C h, \varepsilon)$ around $x_{0}$, we find from A.6 and (6.7),

$$
\begin{aligned}
\varepsilon^{2}\left\|v_{h}\right\|_{H^{1}}^{2} & \leqslant\left(\phi, v_{h}\right) \leqslant C\|\phi\|_{L_{1}}\left\|v_{h}\right\|_{L_{\infty}} \\
& \leqslant C h \ln ^{1 / 2}\left(C+\frac{\varepsilon}{h}\right)\left(\left\|v_{h}\right\|_{H^{\prime}\left(\Omega^{\prime}\right)}+\frac{1}{\varepsilon}\left\|v_{h}\right\|_{\Omega^{\prime}}\right) \\
& \leqslant C h \ln ^{1 / 2}\left(C+\frac{\varepsilon}{h}\right)\left\|v_{h}\right\|_{H^{1}}+\frac{C h^{2}}{\varepsilon^{2}} \ln ^{1 / 2}\left(C+\frac{\varepsilon}{h}\right) .
\end{aligned}
$$

By this,

$$
\left\|v_{h}\right\|_{H^{1}} \leqslant \frac{C h}{\varepsilon^{2}} \ln ^{1 / 2}\left(C+\frac{\varepsilon}{h}\right)
$$

Since also, by Lemmas 4.1 and $2.1,\left\|v-v_{h}\right\|_{H^{1}} \leqslant C h\|v\|_{H^{2}} \leqslant C h / \varepsilon^{2}$, we have completed the proof of the lemma.

We return now to (6.5). Using A.1 (applied to gradients) we infer, via (6.1) and (6.3),

$$
\begin{aligned}
& \left(u-u_{h}, \phi\right)=A_{\varepsilon}\left(u-u_{h}, v\right)=A_{\varepsilon}\left(u-u_{h}, v-v_{h}\right)=A_{\varepsilon}\left(u, v-v_{h}\right) \\
& \quad=-\varepsilon^{2} \sum_{i}\left(\int_{\tau_{i}^{h}} u \Delta\left(v-v_{h}\right)+\oint_{\partial \tau_{i}^{h}} u \frac{\partial}{\partial n}\left(v-v_{h}\right)\right)+\left(a u, v-v_{h}\right) \\
& \quad \leqslant C\|u\|_{L_{\infty}}\left\{\varepsilon^{2}\left|v-v_{h}\right|_{W_{1}^{2, h}}+\varepsilon^{2} h^{-1}\left\|\nabla\left(v-v_{h}\right)\right\|_{L_{1}}+\left\|v-v_{h}\right\|_{L_{1}}\right\}
\end{aligned}
$$

cf. (3.2) for notation. Here, with $\chi$ as in A.4,

$$
\begin{aligned}
\left|v-v_{h}\right|_{W_{1}^{2, h}} & \leqslant|v-\chi|_{W_{1}^{2, h}}+\left|\chi-v_{h}\right|_{W_{1}^{2, h}} \\
& \leqslant C\|v\|_{W_{1}^{2}}+C h^{-1}\left\|\chi-v_{h}\right\|_{W_{1}^{1}} \\
& \leqslant C\|v\|_{W_{1}^{2}}+C h^{-1}\|v-\chi\|_{W_{1}^{1}}+C h^{-1}\left\|v-v_{h}\right\|_{W_{1}^{1}} \\
& \leqslant C\|v\|_{W_{1}^{2}}+C h^{-1}\left\|v-v_{h}\right\|_{W_{1}^{1}} .
\end{aligned}
$$

Therefore, from (6.10),

$$
\begin{aligned}
& \left|h^{-1}\left(u-u_{h}, \phi\right)\right| \\
& \quad \leqslant C\|u\|_{L_{\infty}}\left\{\varepsilon^{2} h^{-1}\|v\|_{W_{1}^{2}}+\varepsilon^{2} h^{-2}\left\|v-v_{h}\right\|_{W_{1}^{1}}+h^{-1}\left\|v-v_{h}\right\|_{L_{1}}\right\} .
\end{aligned}
$$

We next introduce some notation. Let

$$
\begin{gathered}
\Omega_{j}=\left\{x \in \Re: 2^{-j} \leqslant\left|x-x_{0}\right| \leqslant 2^{-j+1}\right\}, \\
\Omega_{j}^{\prime}=\Omega_{j-1} \cup \Omega_{j} \cup \Omega_{j+1}, \quad \Omega_{j}^{\prime \prime}=\Omega_{j-1}^{\prime} \cup \Omega_{j}^{\prime} \cup \Omega_{j}^{\prime} .
\end{gathered}
$$

Assume, for simplicity in writing, that $\Re=\overline{\bigcup_{j=1}^{\infty}} \Omega_{j}$. Let $J_{*}$ be such that $2^{-J_{*}} \leqslant$ $C_{*} h \leqslant 2^{-J_{*}+1}$, with $C_{*}$ sufficiently large, to be determined, and let $\Omega_{*}=\{x \in R$ : $\left.\left|x-x_{0}\right| \leqslant 2^{-J_{*}}\right\}$ so that $\Re=\left(\cup_{j=1}^{J_{*}} \Omega_{j}\right) \cup \Omega_{*}$. Set further $d_{j}=2^{-j}, d_{*}=2^{-J_{*}}$. Also, the notation $\Sigma^{*}\|v\|_{W_{p}^{k}\left(\Omega_{j}\right)}$ shall mean $\sum_{j=1}^{J_{*}}\|v\|_{W_{p}^{k}\left(\Omega_{j}\right)}+\|v\|_{W_{p}^{k}\left(\Omega_{*}\right)}$. 
Returning now to (6.11), we shall estimate the three terms on the right. For the first we have, by Cauchy's inequality and using Lemmas 2.1 and 2.3,

$$
\begin{aligned}
\varepsilon^{2} h^{-1}\|v\|_{W_{1}^{2}} & =\varepsilon^{2} h^{-1} \Sigma^{*}\|v\|_{W_{1}^{2}\left(\Omega_{j}\right)} \leqslant C \varepsilon^{2} h^{-1} \Sigma^{*} d_{j}\|v\|_{H^{2}\left(\Omega_{j}\right)} \\
& \leqslant C+C \varepsilon^{2} h^{-1} \sum_{j=1}^{J_{*}}\|\nabla v\|_{\Omega_{j}^{\prime}}+C h^{-1} \sum_{j=1}^{J_{*}} d_{j}\|v\|_{\Omega_{j}^{\prime}}
\end{aligned}
$$

We note now that

$$
\left(\sum_{j=1}^{J_{*}} e^{-2 c d_{j} / \varepsilon}\right)^{1 / 2} \leqslant \ln ^{1 / 2}\left(C+\frac{\varepsilon}{h}\right) .
$$

For, since $\exp \left(-2 c 2^{-x} / \varepsilon\right)$ is increasing with $x$, we may estimate the sum inside the square root by $I=\int_{1}^{J_{*}+1} \exp \left(-2 c 2^{-x} / \varepsilon\right) d x$. Substituting $2^{-x} / \varepsilon=y$ we have, since $2^{-J_{*}} \leqslant C_{*} h \leqslant 2^{-J_{*}+1}$,

$$
I \leqslant \log _{2} \int_{C_{*} h / 4 \varepsilon}^{1 / 2 \varepsilon} e^{-2 c y} \frac{d y}{y}
$$

and the result readily follows.

Hence, by the exponential decay result of Lemma 2.2 and by Lemma 6.1, for $C_{*}$ large enough,

$$
\begin{aligned}
\varepsilon^{2} h^{-1} \sum_{j=1}^{J_{*}}\|\nabla v\|_{\Omega_{j}^{\prime}} & \leqslant C \varepsilon^{2} h^{-1} \sum_{j=1}^{J_{*}} e^{-c d_{j} / \varepsilon}\|\nabla v\|_{\Omega_{j}^{\prime \prime}} \\
& \leqslant C \varepsilon^{2} h^{-1}\left(\sum_{j=1}^{J_{*}} e^{-2 c d_{j} / \varepsilon}\right)^{1 / 2}\|\nabla v\| \leqslant \ln \left(C+\frac{\varepsilon}{h}\right)
\end{aligned}
$$

Introducing the notation

$$
\mathcal{T} \equiv h^{-1} \Sigma^{*} d_{j}\left(\|v\|_{\Omega_{j}}+\left\|v_{h}\right\|_{\Omega_{j}}\right)
$$

we thus infer from (6.12) and (6.13),

$$
\varepsilon^{2} h^{-1}\|v\|_{W_{1}^{2}} \leqslant C \varepsilon^{2} h^{-1} \Sigma^{*} d_{j}\|v\|_{H^{2}\left(\Omega_{j}\right)} \leqslant \ln \left(C+\frac{\varepsilon}{h}\right)+C \mathcal{J} .
$$

For the third term on the right of (6.11), we immediately see that it is bounded by $C \mathcal{T}$. Therefore, by (6.15),

$$
\left|h^{-1}\left(u-u_{h}, \phi\right)\right| \leqslant C\|u\|_{L_{\infty}}\left\{\ln \left(C+\frac{\varepsilon}{h}\right)+\mathcal{T}+\varepsilon^{2} h^{-2}\left\|v-v_{h}\right\|_{W_{1}^{\prime}}\right\} .
$$

For the last term here, we use the local energy error estimate of Lemma 5.2 and find, for any $\chi \in \delta_{h}$,

$$
\begin{aligned}
\varepsilon^{2} h^{-2}\left\|v-v_{h}\right\|_{W_{1}^{\prime}} \leqslant & C \varepsilon^{2} h^{-2} \Sigma^{*} d_{j}\left\|v-v_{h}\right\|_{H^{1}\left(\Omega_{j}\right)} \\
\leqslant & C \varepsilon^{2} h^{-2} \Sigma^{*}\left(d_{j}\|v-\chi\|_{H^{\prime}\left(\Omega_{j}^{\prime}\right)}+\|v-\chi\|_{\Omega_{j}^{\prime}}\right) \\
& +C \varepsilon^{2} h^{-2} \Sigma^{*} e^{-c d_{j} /(\varepsilon+h)}\left\|v-v_{h}\right\|_{\Omega_{j}^{\prime}} \equiv \Sigma_{1}^{*}+\Sigma_{2}^{*} .
\end{aligned}
$$

Here, by the local approximation hypothesis $A .3$ and by (6.15)

$$
\Sigma_{1}^{*} \leqslant C \varepsilon^{2} h^{-1} \Sigma^{*} d_{j}\|v\|_{H^{2}\left(\Omega_{j}^{\prime \prime}\right)} \leqslant \ln \left(C+\frac{\varepsilon}{h}\right)+C \sigma .
$$


Further, using Lemmas 4.1, and 2.2, 2.1,

$$
\begin{aligned}
\Sigma_{2}^{*} & \leqslant C \varepsilon^{2} h^{-2}\left(\sum e^{-2 c d_{j} /(\varepsilon+h)}\right)^{1 / 2}\left\|v-v_{h}\right\| \\
& \leqslant C \varepsilon^{2} \ln ^{1 / 2}\left(C+\frac{\varepsilon+h}{h}\right)\|v\|_{H^{2}} \leqslant \ln ^{1 / 2}\left(C+\frac{\varepsilon}{h}\right) .
\end{aligned}
$$

Reporting (6.19) and (6.18) into (6.17), and the result of that operation into (6.16),

$$
\left|h^{-1}\left(u-u_{h}, \phi\right)\right| \leqslant C\|u\|_{L_{\infty}}\left\{\ln \left(C+\frac{\varepsilon}{h}\right)+\mathcal{T}\right\} .
$$

It remains to show that

$$
\mathcal{T} \equiv h^{-1} \Sigma^{*} d_{j}\left(\|v\|_{\Omega_{j}}+\left\|v_{h}\right\|_{\Omega_{j}}\right) \leqslant C .
$$

Admitting this for a moment, we would then have

$$
\left|h^{-1}\left(u-u_{h}, \phi\right)\right| \leqslant C\|u\|_{L_{\infty}} \ln \left(C+\frac{\varepsilon}{h}\right)
$$

so that from $(6.4),(6.5)$,

$$
\left\|u-u_{h}\right\|_{L_{\infty}} \leqslant \ln \left(C+\frac{\varepsilon}{h}\right)\|u\|_{L_{\infty}} .
$$

Writing $u-u_{h}=(u-\chi) .-\left(u_{h}-\chi\right)$ for $\chi \in \mathcal{S}_{h}$ would then conclude the proof.

To verify (6.21) we proceed as follows. Since $\|v\|+\left\|v_{h}\right\| \leqslant C$ it suffices to estimate

$$
h^{-1} \sum_{j=1}^{J_{*}} d_{j}\left(\|v\|_{\Omega_{j}}+\left\|v_{h}\right\|_{\Omega_{j}}\right) \text {. }
$$

For $C_{*}$ large enough we may again invoke exponential decay results, now from Lemmas 2.2 and 5.1. Thus,

$$
\begin{aligned}
\sigma \leqslant & C+C h^{-1} \sum_{j=1}^{J_{*}} d_{j} e^{-c d_{j} / \varepsilon}\|v\|_{\Omega_{j}^{\prime}}+C h^{-1} \sum_{j=1}^{J_{*}} d_{j} e^{-c d_{j} /(\varepsilon+h)}\left\|v_{h}\right\|_{\Omega_{j}^{\prime}} \\
\leqslant & C+C h^{-1} \varepsilon\left(\sum_{j=1}^{J_{*}}\left(\frac{d_{j}}{\varepsilon}\right)^{2} e^{-2 c d_{j} / \varepsilon}\right)^{1 / 2}\|v\| \\
& +C h^{-1}(\varepsilon+h)\left(\sum_{j=1}^{J_{*}}\left(\frac{d_{j}}{\varepsilon+h}\right)^{2} e^{-2 c d_{j} /(\varepsilon+h)}\right)^{1 / 2}\left\|v_{h}\right\| \\
\leqslant & C+C h^{-1} \varepsilon\|v\|+C h^{-1}(\varepsilon+h)\left\|v_{h}\right\| .
\end{aligned}
$$

Applying Lemma 6.1 and separating the cases $\varepsilon \leqslant h$ and $\varepsilon \geqslant h$, we deduce (6.21).

This completes the proof of the theorem.

7. A Pointwise Local Error Estimate. We shall establish the following result.

THEOREM 7.1. Let $x_{0} \in \Re$, with (1.2), let $D$ be a disc of radius $d>0$ around it, and set $\Omega_{d}=D \cap \Re$. Let $u$ be a continuous function, with $u=0$ on $\partial \Re$ in the Dirichlet case. Assume that A.1-A.6 of Section 3 hold. Let $u_{h} \in \mathcal{S}_{h}\left(\Omega_{d}\right)$ be such that

$$
A_{\varepsilon}\left(u-u_{h}, \chi\right)=0 \text { for } \chi \in \mathcal{S}_{h}^{\Varangle}\left(\Omega_{d}\right) \text {. }
$$


Assume further that (1.4) holds on $\Omega_{d}$. Then there exist positive constants $c_{1}, c_{2}$ and $C$, independent of $\varepsilon, h, u, u_{h}, x_{0}$ and $d$, such that, for $d \geqslant c_{1} h, 0<\varepsilon \leqslant 1,0<h \leqslant \frac{1}{2}$,

$$
\begin{aligned}
\left|\left(u-u_{h}\right)\left(x_{0}\right)\right| \leqslant C \ln ^{1 / 2}\left(\frac{d}{h}\right)\left\{\ln \left(C+\frac{\varepsilon}{h}\right)\right. & \min _{\chi \in S_{h}}\|u-\chi\|_{L_{\infty}\left(\Omega_{d}\right)} \\
& \left.+\frac{1}{d} e^{-c_{2} d /(\varepsilon+h)}\left\|u-u_{h}\right\|_{L_{2}\left(\Omega_{d}\right)}\right\} .
\end{aligned}
$$

Proof. First change the form $A_{\varepsilon}$ so that (1.4) holds on $R$, with the same $a_{0}$ and $a_{1}$ as for $\Omega_{d}$. This is easily done.

Let $D^{\prime \prime}$ and $D^{\prime}$ be discs concentric with $D$ and of radii $1 / 4 d$ and $1 / 2 d$, respectively, and set $\Omega^{\prime \prime}=\Re \cap D^{\prime \prime}, \Omega^{\prime}=\Re \cap D^{\prime}$. Let $\omega \in \mathcal{C}_{0}^{\infty}(D)$ be such that

$$
\omega \equiv 1 \text { on } D^{\prime}, \quad\|\omega\|_{L_{\infty}(D)} \leqslant D .
$$

Set finally $\tilde{u}=\omega u$ and $\tilde{u}_{h}=P_{h}^{\varepsilon}(\omega u)$. Note that, in the Dirichlet case, $\tilde{u}=0$ on $\partial \Re$. Then

$$
\left|\left(u-u_{h}\right)\left(x_{0}\right)\right|=\left|\left(u-u_{h}\right)\left(x_{0}\right)\right| \leqslant\left|\left(\tilde{u}-\tilde{u}_{h}\right)\left(x_{0}\right)\right|+\left|\left(\tilde{u}_{h}-u_{h}\right)\left(x_{0}\right)\right| .
$$

Here, by Theorem 6.1,

$$
\left|\left(\tilde{u}-\tilde{u}_{h}\right)\left(x_{0}\right)\right| \leqslant \ln \left(C+\frac{\varepsilon}{h}\right)\|\tilde{u}\|_{L_{\infty}} \leqslant \ln \left(C+\frac{\varepsilon}{h}\right)\|u\|_{L_{\infty}\left(\Omega_{d}\right)} .
$$

For the function $\tilde{u}_{h}-u_{h}$ in $\delta_{h}\left(\Omega_{d}\right)$ we have by (7.3),

$$
A_{\varepsilon}\left(\tilde{u}_{h}-u_{h}, \chi\right)=0 \text { for } \chi \in \mathcal{S}_{h}^{\Varangle}\left(\Omega^{\prime}\right) \text {. }
$$

Using then the Sobolev inequality A.6 and Lemma 5.1, and also (7.3),

$$
\begin{aligned}
\left|\left(\tilde{u}_{h}-u_{h}\right)\left(x_{0}\right)\right| \leqslant C(\ln d / h)^{1 / 2}\left\{\left\|\tilde{u}_{h}-u_{h}\right\|_{H^{\prime}\left(\Omega^{\prime \prime}\right)}+\frac{1}{d}\left\|\tilde{u}_{h}-u_{h}\right\|_{\Omega^{\prime \prime}}\right\} \\
\leqslant \frac{C}{d}(\ln d / h)^{1 / 2} e^{-c d /(\varepsilon+h)}\left\|\tilde{u}_{h}-u_{h}\right\|_{\Omega^{\prime}} \\
\leqslant \frac{C}{d}(\ln d / h)^{1 / 2}\left\|\tilde{u}_{h}-\tilde{u}\right\|_{\Omega^{\prime}}+\frac{C(\ln d / h)^{1 / 2}}{d} e^{-c d /(\varepsilon+h)}\left\|u-u_{h}\right\|_{\Omega^{\prime}}
\end{aligned}
$$

Here the first term on the right is bounded, using again Theorem 6.1, as

$$
C(\ln d / h)^{1 / 2}\left\|\tilde{u}_{h}-\tilde{u}\right\|_{L_{\infty}} \leqslant \ln \left(C+\frac{\varepsilon}{h}\right) \ln (d / h)^{1 / 2}\|u\|_{L_{\infty}\left(\Omega_{d}\right)} .
$$

Combining the above we have proven (7.2) with $\chi=0$. Writing $u-u_{h}=(u-\chi)$ $-\left(u_{h}-\chi\right)$ for $\chi \in \mathcal{S}_{h}$ verifies it as stated.

8. Outline of the One-Dimensional Case. We consider the problem of finding $u=u(x)$ such that

$$
-\varepsilon^{2} u^{\prime \prime}+a(x ; \varepsilon) u=f \text { in } \Re=[I, J] \text {, a finite interval, } u \in \mathscr{B},
$$

where the analogue of (1.4) holds and where $u \in \mathscr{B}$ designates homogeneous Dirichlet or Neumann conditions at $x=I$ and $x=J$. In the present case there is no additional work incurred by allowing different boundary conditions at the two endpoints.

The finite element spaces $\varsigma_{h}$ typically consist of piecewise polynomials of a certain degree on a quasi-uniform mesh, the polynomials on different subintervals being 
connected by continuity constraints across nodes. For a Dirichlet condition at $x=I$ or $J$, it is assumed that the functions in $\widehat{S}_{h}$ vanish there.

The analogues of hypotheses A.1-A.5 of Section 3 are easy to verify in practice; A.6 will not be needed in the one-dimensional situation. In A.2, the factor in front of $\left(q^{-1}-p^{-1}\right)$ is 1 instead of 2 .

The results of Sections 2, 4 and 5 have no changes in statement; their proofs are often somewhat simpler, especially in Section 2.

We shall now outline how Theorem 6.1 would be proved, with (6.2) replaced by

$$
\left\|u-u_{h}\right\|_{L_{\infty}(\Re)} \leqslant C \min _{\chi \in \delta_{h}}\|u-\chi\|_{L_{\infty}(\Re)} .
$$

For another approach to the same result, see Wahlbin [27]. (The corresponding local result is given in (8.3) below.) We have, referring to analogous estimates in Section 6,

$$
\left|\left(u-u_{h}\right)\left(x_{0}\right)\right| \leqslant C\|u\|_{L_{\infty}}+C h^{-1 / 2}\left\|u-u_{h}\right\|_{\tau_{0}} .
$$

One then proceeds as in (6.5), (6.6). Lemma 6.1 will now have the following inequalities:

$$
\begin{gathered}
\|v\|+\left\|v_{h}\right\| \leqslant\left\{\begin{array}{l}
C h^{1 / 2} / \varepsilon^{1 / 2}, \\
C,
\end{array}\right. \\
\left\|v^{\prime}\right\|+\left\|v_{h}^{\prime}\right\| \leqslant C h^{1 / 2} / \varepsilon^{3 / 2} .
\end{gathered}
$$

To see this, consider, e.g., $v$. By use of the one-dimensional Sobolev inequality $\|g\|_{L_{\infty}} \leqslant C\left(\left\|g^{\prime}\right\|^{1 / 2}\|g\|^{1 / 2}+\|g\|\right)$, we have

$$
\begin{aligned}
\varepsilon^{2}\left\|v^{\prime}\right\|^{2}+a_{0}\|v\|^{2} & \leqslant(\phi, v) \leqslant\|\phi\|_{L_{1}}\|v\|_{L_{\infty}} \\
& \leqslant C h^{1 / 2}\|\phi\|_{L_{2}}\left(\|v\|^{1 / 2}\left\|v^{\prime}\right\|^{1 / 2}+\|v\|\right) \\
& \leqslant C\left(\frac{h^{1 / 2}}{\varepsilon^{1 / 2}}\|v\|^{1 / 2}\right)\left(\varepsilon^{1 / 2}\left\|v^{\prime}\right\|^{1 / 2}\right)+C h^{1 / 2}\|v\| \\
& \leqslant C\left(\frac{h^{1 / 2}}{\varepsilon^{1 / 2}}\|v\|^{1 / 2}\right)^{4 / 3}+\frac{1}{2} \varepsilon^{2}\left\|v^{\prime}\right\|^{2}+C h+\frac{a_{0}}{2}\|v\|^{2}
\end{aligned}
$$

Thus

$$
\frac{a_{0}}{2}\|v\|^{2} \leqslant C\left(h^{1 / 2} \varepsilon^{-1 / 2}\|v\|^{1 / 2}\right)^{4 / 3}+h
$$

from which (half of) $(6.7)^{\prime}$ follows. The proofs of $(6.7)^{\prime}$ and $(6.8)^{\prime}$ are easily completed.

Now continue as in Section 6. This time,

$(6.11)^{\prime}\left|h^{-1 / 2}\left(u-u_{h}, \phi\right)\right|$

$$
\leqslant C\|u\|_{L_{\infty}}\left\{\varepsilon^{2} h^{-1 / 2}\|v\|_{W_{1}^{2}}+\varepsilon^{2} h^{-3 / 2}\left\|v-v_{h}\right\|_{W_{1}^{1}}+h^{-1 / 2}\left\|v-v_{h}\right\|_{L_{1}}\right\} .
$$

Here, trivially from the equation $L_{\varepsilon} v=\phi$,

$$
\varepsilon^{2} h^{-1 / 2}\|v\|_{W_{1}^{2}} \leqslant h^{-1 / 2}\|\phi\|_{L_{1}}+h^{-1 / 2} a_{1}\|v\|_{L_{1}} \leqslant C+h^{-1 / 2} a_{1}\|v\|_{L_{1}} .
$$

Setting now

$$
\mathcal{T}^{\prime} \equiv h^{-1 / 2} \Sigma^{*} d_{j}^{1 / 2}\left(\|v\|_{\Omega_{j}}+\left\|v_{h}\right\|_{\Omega_{j}}\right),
$$


we immediately see that $h^{-1 / 2}\|v\|_{L_{1}} \leqslant C^{\mathcal{T}^{\prime}}$. Also the last term on the right of (6.11)' is bounded by $C^{\sigma^{\prime}}$. Thus,

(6.16) $\left|h^{-1 / 2}\left(u-u_{h}, \phi\right)\right| \leqslant C\|u\|_{L_{\infty}}\left\{1+\mathcal{T}^{\prime}+\varepsilon^{2} h^{-3 / 2}\left\|v-v_{h}\right\|_{W_{1}^{\prime}}\right\}$.

Using the local energy error estimates of Lemma 5.2, we find $(6.17)^{\prime} \quad \varepsilon^{2} h^{-3 / 2}\left\|v-v_{h}\right\|_{W_{1}^{1}} \leqslant \varepsilon^{2} h^{-3 / 2} \Sigma^{*} d_{j}^{1 / 2}\left\|v-v_{h}\right\|_{H^{\prime}\left(\Omega_{j}\right)}$

$$
\begin{aligned}
\leqslant & C \varepsilon^{2} h^{-3 / 2} \Sigma^{*}\left(d_{j}^{1 / 2}\|v-\chi\|_{H^{1}\left(\Omega_{j}^{\prime}\right)}+d_{j}^{-1 / 2}\|v-\chi\|_{\Omega_{j}^{\prime}}\right) \\
& +C \varepsilon^{2} h^{-3 / 2} \Sigma^{*} d_{j}^{-1 / 2}\left\|v-v_{h}\right\|_{\Omega_{j}^{\prime}} \equiv \Sigma_{1}^{*}+\Sigma_{2}^{*}
\end{aligned}
$$

Here, by local approximation, A.3, and since $\varepsilon v^{\prime \prime}=a v$ outside $\Omega_{*}$, using also Lemma 2.1,

$$
\begin{aligned}
\Sigma_{1}^{*} & \leqslant C \varepsilon^{2} h^{-1 / 2} \Sigma^{*} d_{j}^{1 / 2}\|v\|_{H^{2}\left(\Omega_{j}^{\prime \prime}\right)} \\
& \leqslant C \varepsilon^{2}\|v\|_{H^{2}}+C h^{-1 / 2} \sum_{j=1}^{\mathcal{T}_{*}^{*}} d_{j}^{1 / 2}\|v\|_{\Omega_{j}^{\prime \prime}} \leqslant C+C \mathcal{T}^{\prime} .
\end{aligned}
$$

Further, by use of Cauchy-Schwarz' inequality and by Lemmas 4.1 and 2.1,

$$
\Sigma_{2}^{*} \leqslant C \varepsilon^{2} h^{-2}\left\|v-v_{h}\right\| \leqslant C \varepsilon^{2}\|v\|_{H^{2}} \leqslant C .
$$

Thus, from (6.16)' via (6.17)' and $(6.18)^{\prime},(6.19)^{\prime}$,

$$
\left|h^{-1 / 2}\left(u-u_{h}, \phi\right)\right| \leqslant C\|u\|_{L_{\infty}}\left\{1+\sigma^{\prime}\right\}
$$

It remains to show that

$$
\mathcal{T}^{\prime} \leqslant C .
$$

This now goes as follows. By use of the exponential decay results and of (6.7)',

$$
\begin{aligned}
\mathscr{T}^{\prime} \leqslant & C+C h^{-1 / 2} \sum_{j=1}^{\mathcal{T}_{*}} d_{j}^{1 / 2} e^{-c d_{j} / \varepsilon}\|v\|_{\Omega_{j}^{\prime}} \\
& +C h^{-1 / 2} \sum_{*}^{\mathcal{T}_{*}} d_{j}^{1 / 2} e^{-c d_{j} /(\varepsilon+h)}\left\|v_{h}\right\|_{\Omega_{j}^{\prime}} \\
\leqslant & C+C h^{-1 / 2} \varepsilon^{1 / 2}\left(\sum_{j} \frac{d_{j}}{\varepsilon} e^{-c d_{j} / \varepsilon}\right)^{1 / 2}\|v\| \\
& +C h^{-1 / 2}(\varepsilon+h)^{1 / 2}\left(\sum_{j} \frac{d_{j}}{(\varepsilon+h)} e^{-c d_{j} /(\varepsilon+h)}\right)^{1 / 2}\left\|v_{h}\right\| \\
\leqslant & C .
\end{aligned}
$$

This completes the proof of (8.2).

For the local result corresponding to Theorem 7.1 , one obtains it with (7.2) replaced by

$$
\begin{aligned}
& \text { (8.3) }\left|\left(u-u_{h}\right)\left(x_{0}\right)\right| \\
& \quad \leqslant C\left\{\min _{\chi \in \delta_{h}}\|u-\chi\|_{L_{\infty}\left(\Omega_{d}\right)}+\frac{1}{\sqrt{d}} e^{-c d /(\varepsilon+h)}\left\|u-u_{h}\right\|_{L_{2}\left(\Omega_{d}\right)}\right\} .
\end{aligned}
$$


To see this one proceeds as in the proof of Theorem 7.1. The inequality (7.4) is as before, whereas, by (8.2),

$$
\left|\left(\tilde{u}-\tilde{u}_{h}\right)\left(x_{0}\right)\right| \leqslant C\|\tilde{u}\|_{L_{\infty}} \leqslant C\|u\|_{L_{\infty}\left(\Omega_{d}\right)} .
$$

In (7.6) we use instead the one-dimensional Sobolev inequality

$$
\left|g\left(x_{0}\right)\right| \leqslant C \sqrt{d}\left\|g^{\prime}\right\|_{\Omega^{\prime \prime}}+\frac{C}{\sqrt{d}}\|g\|_{\Omega^{\prime \prime}}
$$

so that, by Lemma 5.1 and (7.3),

$$
\begin{aligned}
&\left|\left(\tilde{u}_{h}-u_{h}\right)\left(x_{0}\right)\right| \leqslant \frac{C}{\sqrt{d}} e^{-c d /(\varepsilon+h)}\left\|\tilde{u}_{h}-u_{h}\right\|_{\Omega^{\prime}} \\
& \leqslant \frac{C}{\sqrt{d}}\left\|\tilde{u}_{h}-\tilde{u}\right\|_{\Omega^{\prime}}+\frac{C}{\sqrt{d}} e^{-c d /(\varepsilon+h)}\left\|u-u_{h}\right\|_{\Omega^{\prime}}
\end{aligned}
$$

The proof is then completed as in Section 7.

9. Nonhomogeneous Boundary Conditions. We shall show how Theorems 6.1 and 7.1 carry over to nonhomogeneous conditions (1.3).

Let us first consider Neumann boundary conditions, i.e., $\partial u / \partial n=F$ on $\partial \Re$. The weak form of (1.1.a) $)_{\text {lin }}$ is to find $u \in H^{1}$ such that with, as before, $A_{\varepsilon}(v, w)=$ $\varepsilon^{2}(\nabla v, \nabla w)+(a v, w)$ and with $\langle v, w\rangle=\int_{\partial \Re} v w d s$, we have

$$
A_{\varepsilon}(u, v)+\varepsilon^{2}\langle F, v\rangle=(f, v) \text { for all } v \in H^{1} .
$$

The Galerkin solution $u_{h}$ is found as $u_{h} \in S_{h}$ (with no imposed boundary condition) such that

$$
A_{\varepsilon}\left(u_{h}, \chi\right)+\varepsilon^{2}\langle F, \chi\rangle=(f, \chi) \text { for } \chi \in S_{h} .
$$

Here we shall not consider further approximation of $\langle F, \chi\rangle$. Hence,

$$
A_{\varepsilon}\left(u-u_{h}, \chi\right)=0 \quad \text { for } \chi \in S_{h}
$$

and the results of Theorems 6.1 and 7.1 are clearly true without any change in statements or proofs.

The case of essential Dirichlet conditions is somewhat more technical. We seek to approximate $u$, the solution of

$$
L_{\varepsilon} u=f \text { in } \Re, \quad u=F \text { on } \partial \Re .
$$

The approximation $u_{h}$ in $S_{h}$ is found via

$$
A_{\varepsilon}\left(u_{h}, \chi\right)=0 \text { for } \chi \in \dot{S}_{h}, \quad u_{h}=F_{h} \text { on } \partial \mathcal{R}
$$

where $\dot{S}_{h}$ denotes those functions in $S_{h}$ that vanish on $\partial \Re$ and where $F_{h}$, ari approximation to $F$, is the restriction to $\partial \mathscr{R}$ of some function in $S_{h}$. Then

$$
A_{\varepsilon}\left(u-u_{h}, \chi\right)=0 \text { for } \chi \in \dot{S}_{h} \text {, where } u_{h}=F_{h} \text { on } \partial \Re \text {. }
$$

The result of Theorem 6.1 now goes as follows: With appropriate notations and conditions from there, if $(9.1)$ holds, then

$$
\left\|u-u_{h}\right\|_{L_{\infty}(\Re)} \leqslant \ln \left(C+\frac{\varepsilon}{h}\right) \min _{\substack{\chi \in S_{h} \\ \chi=u_{h} \text { on } \partial \Re}}\|u-\chi\|_{L_{\infty}(\Re)} .
$$


For the localization of this we have, with notations and conditions taken from Theorem 7.1 (but not repeated here): If $u_{h} \in S_{h}\left(\Omega_{d}\right)$ with (in self explanatory notation)

$$
A_{\varepsilon}\left(u-u_{h}, \chi\right)=0 \text { for } \chi \in S_{h}^{\ddagger} \Varangle\left(\Omega_{d}\right),
$$

then

$$
\begin{aligned}
& \left|\left(u-u_{h}\right)\left(x_{0}\right)\right| \leqslant \ln \left(C+\frac{\varepsilon}{h}\right) \ln ^{1 / 2}\left(\frac{d}{h}\right) \\
& \quad \times\left\{\min _{\substack{x \in S_{h}\left(\Omega_{d}\right) \\
\chi=u_{h} \text { on } \Omega_{d} \cap \partial \Re}}\|u-\chi\|_{L_{\infty}\left(\Omega_{d}\right)}+\frac{1}{d} e^{-c_{2} d /(\varepsilon+h)}\left\|u-u_{h}\right\|_{L_{2}\left(\Omega_{d}\right)}\right\} .
\end{aligned}
$$

In the rest of this section we shall show how proving (9.4) can be reduced to the previous case of a homogeneous Dirichlet condition. The analysis leading to the global estimate (9.2) is similar and simpler and we leave it to the reader.

By a density argument, we may assume that $u \in \mathcal{C}^{2}\left(\Omega_{d}\right)$. Set $\Gamma=\Omega_{d} \cap \partial \Re$; we shall assume $\Gamma$ nonempty and leave the (much easier) case of empty $\Gamma$ to the reader. Designate $F=\left.u\right|_{\Gamma}$, the boundary values of $u$, and $F_{h}=\left.u_{h}\right|_{\Gamma}$.

We may assume that the circle of radius $d$ around $x_{0}$ cuts $\partial \Re$ in two points only; otherwise use smaller but comparable discs. First, extend $F-F_{h}$ from $\Gamma$ to $\partial \Omega_{d}$ so that

$$
\left\|F-F_{h}\right\|_{L_{\infty}\left(\partial \Omega_{d}\right)}=\left\|F-F_{h}\right\|_{L_{\infty}(\Gamma)}
$$

this is easily done since only two values of $F-F_{h}$ are given on the circular arc of $\partial \Omega_{d} \backslash \Gamma$.

Define next $\psi$ by

$$
L_{\varepsilon}(\psi-u)=0 \quad \text { in } \Omega_{d}, \quad \psi-u=F_{h}-F \quad \text { on } \partial \Omega_{d} .
$$

Then

$$
\psi=F_{h} \text { on } \Gamma
$$

and, by the maximum principle and (9.5),

$$
\|\psi-u\|_{L_{\infty}\left(\Omega_{d}\right)} \leqslant\left\|F-F_{h}\right\|_{L_{\infty}(\Gamma)} .
$$

Hence,

$$
\begin{aligned}
\left|\left(u-u_{h}\right)\left(x_{0}\right)\right| & \leqslant\left|(u-\psi)\left(x_{0}\right)\right|+\left|\left(\psi-u_{h}\right)\left(x_{0}\right)\right| \\
& \leqslant\left\|F-F_{h}\right\|_{L_{\infty}(\Gamma)}+\left|\left(\psi-u_{h}\right)\left(x_{0}\right)\right|
\end{aligned}
$$

Here,

$$
A_{\varepsilon}\left(\psi-u_{h}, \chi\right)=0 \text { for } \chi \in \dot{S}_{h}^{\Varangle}\left(\Omega_{d}\right) .
$$

Let $\phi_{h}$ be any function in $S_{h}\left(\Omega_{d}\right)$ with $\phi_{h}=F_{h}$ on $\Gamma$, and set

$$
\eta=\psi-\phi_{h}, \quad \theta=u_{h}-\phi_{h} \text {. }
$$


Note that $\eta=0$ on $\Gamma$ and that $\theta \in \stackrel{\circ}{h}_{h}\left(\Omega_{d}\right)$. Since, by $(9.9), A_{\varepsilon}(\eta-\theta, \chi)=0$ for $\chi \in S_{h}^{\ddagger}\left(\Omega_{d}\right)$, we have from Theorem 7.1

$$
\begin{aligned}
\left|\left(\psi-u_{h}\right)\left(x_{0}\right)\right|= & \left|(\eta-\theta)\left(x_{0}\right)\right| \leqslant \ln \left(C+\frac{\varepsilon}{h}\right) \ln ^{1 / 2}\left(\frac{d}{h}\right) \\
& \times\left\{\|\eta\|_{L_{\infty}\left(\Omega_{d}\right)}+\frac{1}{d} e^{-c_{2} d /(\varepsilon+h)}\|\eta-\theta\|_{L_{2}\left(\Omega_{d}\right)}\right\} .
\end{aligned}
$$

Here, by (9.7),

$$
\begin{aligned}
\|\eta\|_{L_{\infty}\left(\Omega_{d}\right)} & =\left\|\psi-\phi_{h}\right\|_{L_{\infty}\left(\Omega_{d}\right)} \\
& \leqslant\|u-\psi\|_{L_{\infty}\left(\Omega_{d}\right)}+\left\|u-\phi_{h}\right\|_{L_{\infty}\left(\Omega_{d}\right)} \\
& \leqslant\left\|F-F_{h}\right\|_{L_{\infty}(\Gamma)}+\left\|u-\phi_{h}\right\|_{L_{\infty}\left(\Omega_{d}\right)} .
\end{aligned}
$$

By a similar argument,

$$
\begin{aligned}
\frac{1}{d}\|\eta-\theta\|_{L_{2}\left(\Omega_{d}\right)} & =\frac{1}{d}\left\|\psi-u_{h}\right\|_{L_{2}\left(\Omega_{d}\right)} \\
& \leqslant \frac{1}{d}\|\psi-u\|_{L_{2}\left(\Omega_{d}\right)}+\frac{1}{d}\left\|u-u_{h}\right\|_{L_{2}\left(\Omega_{d}\right)} \\
& \leqslant \sqrt{\pi}\left\|F-F_{h}\right\|_{L_{\infty}(\Gamma)}+\frac{1}{d}\left\|u-u_{h}\right\|_{L_{2}\left(\Omega_{d}\right)} .
\end{aligned}
$$

Using (9.12) and (9.11) in (9.10) and combining with (9.8) shows that

$$
\begin{aligned}
\left|\left(u-u_{h}\right)\left(x_{0}\right)\right| \leqslant & \ln \left(C+\frac{\varepsilon}{h}\right) \ln ^{1 / 2}\left(\frac{d}{h}\right) \\
& \times\left\{\left\|F-F_{h}\right\|_{L_{\infty}(\Gamma)}+\left\|u-\phi_{h}\right\|_{L_{\infty}\left(\Omega_{d}\right)}\right. \\
& \left.+\frac{1}{d} e^{-c_{2} d /(\varepsilon+h)}\left\|u-u_{h}\right\|_{L_{2}\left(\Omega_{d}\right)}\right\} .
\end{aligned}
$$

Since $u=F, u_{h}=\phi_{h}=F_{h}$ on $\Gamma$, where $\phi_{h}$ is otherwise arbitrary in $S_{h}\left(\Omega_{d}\right)$,

$$
\left\|F-F_{h}\right\|_{L_{\infty}(\Gamma)}+\left\|u-\phi_{h}\right\|_{L_{\infty}\left(\Omega_{d}\right)} \leqslant 2 \min _{\substack{\chi \in S_{h}\left(\Omega_{d}\right) \\ \chi=u_{h} \text { on } \Omega_{d} \cap \partial \Re}}\|u-\chi\|_{L_{\infty}\left(\Omega_{d}\right)} .
$$

This proves (9.4) under the condition (9.3).

We leave the translation of the one-dimensional estimates to the case of nonhomogeneous boundary conditions to the reader.

10. More Explicit Local Error Estimates. In this section we shall explicate the local estimate (1.8) under certain smoothness assumptions effectively reducing the nonsmoothness in the problem to the boundary layer. We shall only consider the homogeneous Dirichlet case.

We make the following three assumptions:

(1) (Local behavior of $u$.) There exist positive constants $C$ and $c$, independent of $\varepsilon$, such that with $d(x)=\operatorname{dist}(x, \partial \Re)$,

$$
\left|\left(\frac{\partial}{\partial x}\right)^{\alpha} u(x)\right| \leqslant C\left(1+\frac{e^{-c d(x) / \varepsilon}}{\varepsilon^{2}}\right) \quad \text { for }|\alpha| \leqslant 2 .
$$


If data in the problem, including $\partial \mathscr{R}$, are sufficiently smooth, the estimate (10.1) is a consequence of Besjes [4, Theorem 13].

(2) There exists a constant $c$ such that

$$
\|f\|_{L_{\infty}(\Re)} \leqslant C \text { independent of } 0<\varepsilon \leqslant 1 .
$$

We note that then (see Lemma A.1 in the Appendix)

$$
\|u\|_{L_{\infty}} \leqslant \frac{1}{a_{0}}\|f\|_{L_{\infty}} \leqslant C .
$$

Finally, we need a local maximum norm approximation estimate for $\dot{S}_{h}$.

(3) There exist constants $c$ and $C$ such that the following holds. Given a function $v$ with $v=0$ on $\partial \Re$ there exists $\chi \in \stackrel{\circ}{S}_{h}$ such that for $0<h \leqslant 1 / 2, i=1, \ldots, I(h)$,

$$
\|v-\chi\|_{L_{\infty}\left(\tau_{i}^{h}\right)} \leqslant C h^{2}\|v\|_{\mathcal{C}^{2}\left(\tilde{\tau}_{i}^{h}\right)}
$$

where $\tilde{\tau}_{i}^{h}=\cup_{\operatorname{dist}\left(\tau_{i}^{h}, \tau_{j}^{h}\right) \leqslant c h} \tau_{j}^{h}$.

Setting $e(x)=u(x)-u_{h}(x)$, the following is the main result of this section.

Theorem 10.1. Assume (1.2), (1.4), A.1-A.6 of Section 3 and (1), (2), (3) above. There exist positive constants $c_{1}, c_{2}, c_{3}$ and $C$, independent of $h$ and $\varepsilon$, such that with $d(x)=\operatorname{dist}(x, \partial \Re)$, the following holds.

(i) If $\varepsilon \leqslant h$, then

$$
|e(x)| \leqslant C \ln ^{1 / 2}\left(\frac{d}{h}\right) h^{2} \quad \text { for } d(x) \geqslant c_{2} h \ln 1 / h
$$

and

$$
|e(x)| \leqslant C \quad \text { for } d(x) \leqslant c_{2} h \ln 1 / h
$$

If furthermore $\varepsilon \leqslant c_{1} h / \ln 1 / h$, then

$$
|e(x)| \leqslant C \ln ^{1 / 2}\left(\frac{d}{h}\right)\left\{h^{2}+e^{-c_{3} d / h}\right\} \quad \text { for } c_{2} h \leqslant d(x) .
$$

(ii) If $\varepsilon \geqslant h$, then

$$
|e(x)| \leqslant \ln ^{1 / 2}\left(\frac{d}{h}\right) \ln \left(C+\frac{\varepsilon}{h}\right) h^{2} \quad \text { for } d(x) \geqslant c_{2} \varepsilon \ln 1 / \varepsilon
$$

and

$$
|e(x)| \leqslant \ln \left(C+\frac{\varepsilon}{h}\right) \frac{h^{2}}{\varepsilon^{2}} \quad \text { for any } d(x)
$$

Remark. In a one-dimensional problem, the factors $\ln ^{1 / 2}(d / h)$ and $\ln (C+\varepsilon / h)$ would not be present.

Before giving the proof of the theorem, we display, in Figure 10.1, the error estimates obtained. Factors $\ln ^{1 / 2}(d / h), \ln (C+\varepsilon / h)$ and constants are skipped. The shaded regions are intended to suggest (small) areas where our information is less precise. 


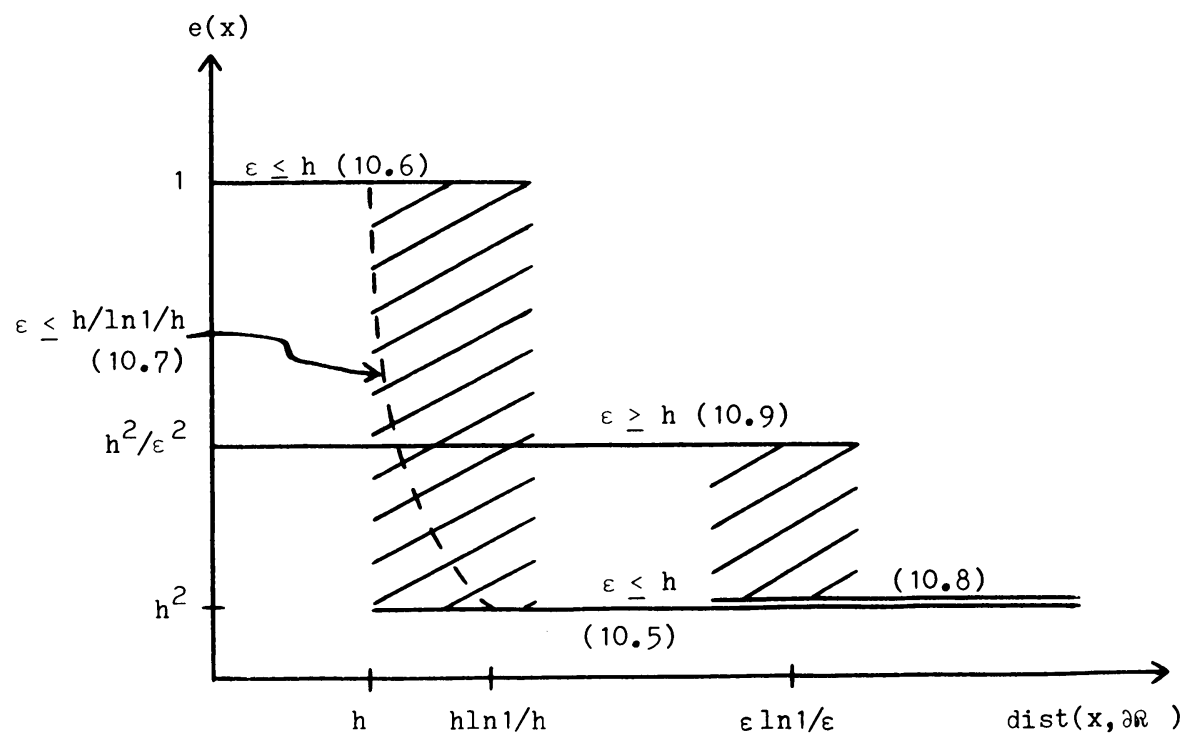

FIGURE 10.1

Local error estimates according to Theorem 10.1

Proof of Theorem 10.1. The easier estimates are (10.6) and (10.9). Repeating (1.7),

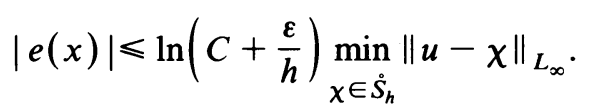

For (10.6), take $\chi \equiv 0$ and use (10.3) and that $\varepsilon / h \leqslant 1$. For (10.9), using (10.4) and (10.1) one has immediately

$$
\min _{\chi \in \dot{S}_{h}}\|u-\chi\|_{L_{\infty}} \leqslant C h^{2}\|u\|_{e^{2}} \leqslant \frac{C h^{2}}{\varepsilon^{2}} .
$$

The remaining results are based on the local estimate (1.8). Taking there $d$ equalling the present $d(x)=\operatorname{dist}(x, \partial \Re)$, we have for $d \geqslant c^{\prime} h$,

$$
\begin{aligned}
\text { (10.11) }|e(x)| \leqslant C \ln ^{1 / 2}\left(\frac{d}{h}\right)\left\{\ln \left(C+\frac{\varepsilon}{h}\right) \min _{x \in \tilde{S}_{h}}\|u-\chi\|_{L_{\infty}\left(\Omega_{d}\right)}\right. \\
\left.+\frac{c}{d} e^{-c^{\prime \prime} d /(\varepsilon+h)}\left\|u-u_{h}\right\|_{L_{2}\left(\Omega_{d}\right)}\right\},
\end{aligned}
$$

where $\operatorname{dist}\left(\Omega_{d}, \partial \Re\right) \geqslant c^{\prime} d / 2$.

Note now the elementary inequality

$$
\frac{e^{-c d / \varepsilon}}{\varepsilon^{2}} \leqslant 1 \quad \text { for } d \geqslant c_{2} \varepsilon \ln 1 / \varepsilon, \quad c c_{2} \geqslant 2 .
$$

We further estimate $d^{-1}\left\|u-u_{h}\right\|_{L_{2}\left(\Omega_{d}\right)}$ by $C\left\|u-u_{h}\right\|_{L_{\infty}} \leqslant \ln (C+\varepsilon / h)$, cf. (10.10) et seq. Using then (10.4) and (10.1) in a local fashion, (10.11) gives for $d \geqslant c^{\prime} h$,

(10.13) $|e(x)| \leqslant C \ln ^{1 / 2}\left(\frac{d}{h}\right) \ln \left(C+\frac{\varepsilon}{h}\right)\left\{h^{2}\left(1+\frac{e^{-c d / \varepsilon}}{\varepsilon^{2}}\right)+e^{-c^{\prime \prime} d /(\varepsilon+h)}\right\}$. 
For $\varepsilon \leqslant h$ and $d \geqslant c_{2} h \ln 1 / h \geqslant c_{2} \varepsilon \ln 1 / \varepsilon,(10.12)$ and a similar argument for the last term give, since $\varepsilon / h \leqslant 1$,

$$
|e(x)| \leqslant C \ln ^{1 / 2}(d / h) h^{2}
$$

for $c c_{2} \geqslant 2, c^{\prime \prime} c_{2} \geqslant 2$. This is (10.5).

For (10.7), one easily finds that $d \geqslant c\left(c_{2}, c_{1}\right) \varepsilon \ln 1 / \varepsilon$, and hence the result again follows from (10.13) and (10.12).

Lastly, (10.8) also follows from (10.13) by similar arguments.

This proves the theorem.

11. Numerical Examples. In this section we shall elucidate some simple numerical examples via our theory, and vice versa.

Example 11.1. Let

$$
u(x)=x-1-x e^{-1 / \varepsilon}+e^{-x / \varepsilon},
$$

so that $u$ solves the problem

$$
\left\{\begin{array}{l}
-\varepsilon^{2} u^{\prime \prime}+u=x-1-x e^{-1 / \varepsilon}, \quad 0 \leqslant x \leqslant 1, \\
u(0)=u(1)=0
\end{array}\right.
$$

The function $u$ is simply a linear term superimposed on a decaying exponential exhibiting, for small $\varepsilon$, a boundary layer of width $\varepsilon \ln 1 / \varepsilon$ at $x=0$. Note that the coefficients and right-hand side in (11.1) are nice functions, uniformly in $0<\varepsilon \leqslant 1$.

We solved this problem numerically for a range of $\varepsilon$ 's, employing uniform meshes of size $h=.05$ and $h=.025$ and, in each of these cases, using piecewise linear and Hermite cubic approximations.

In Table 11.1 we exhibit the maximum norm error (determined at meshpoints only) and the meshpoint $j h$ where it occured.

TABLE 11.1

Maximum error for a range of $\varepsilon$ 's

\begin{tabular}{|l|c|c|c|r|}
\cline { 2 - 5 } & \multicolumn{4}{|c|}{ PIECEWISE LINEARS } \\
\cline { 2 - 5 }$\varepsilon$ & $h=.05$ & $j$ & $h=.025$ & $j$ \\
\cline { 2 - 5 } $5^{0}$ & $.15-4$ & 9 & $.37-5$ & 18 \\
$5^{-1}$ & $.96-3$ & 4 & $.24-3$ & 8 \\
$5^{-2}$ & $.27-1$ & 1 & $.60-2$ & 2 \\
$5^{-3}$ & .21 & 1 & .12 & 1 \\
$5^{-4}$ & .26 & 1 & .26 & 1 \\
$5^{-5}$ & .27 & 1 & .27 & 1 \\
$5^{-6}$ & .27 & 1 & .27 & 1 \\
\hline
\end{tabular}

\begin{tabular}{|c|c|c|c|}
\hline \multicolumn{4}{|c|}{ HERMITE CUBICS } \\
\hline$h=.05$ & $j$ & $h=.025$ & $j$ \\
\hline $.76-8$ & 2 & $.50-9$ & 3 \\
$.34-5$ & 1 & $.26-6$ & 2 \\
$.83-3$ & 1 & $.90-4$ & 1 \\
$.33-1$ & 1 & $.94-2$ & 1 \\
$.78-1$ & 1 & $.68-1$ & 1 \\
$.82-1$ & 1 & $.82-1$ & 1 \\
$.82-1$ & 1 & $.82-1$ & 1 \\
\hline
\end{tabular}

We shall now investigate the cases $\varepsilon \gg h, \varepsilon \sim h$ and $\varepsilon \ll h$ in more detail.

$\varepsilon \gg h$. For $\varepsilon$ large compared to $h$, the estimates (10.8) and (10.9) show that we may expect the global maximum norm error to behave like $O\left(h^{2} / \varepsilon^{2}\right)$, for piecewise linears. In the case of Hermite cubics, since we know $u(x)$ explicitly, it is easy to derive an $O\left(h^{4} / \varepsilon^{4}\right)$ estimate from (1.7). Computing global rates of convergence from Table 11.1, we find them as in Table 11.2. 
TABle 11.2

Computed rates of convergence ( global)

\begin{tabular}{|c|c|c|}
\hline$\varepsilon$ & Piec. Lin. & Herm. Cub. \\
\hline $5^{0}$ & 2.0 & 3.9 \\
$5^{-1}$ & 2.0 & 3.7 \\
$5^{-2}$ & 2.2 & 3.2 \\
\hline
\end{tabular}

For smaller $\varepsilon$, the computed global rates deviate considerably from 2 and 4 . In particular, for very low $\varepsilon$, there is no decrease in error with $h$.

$\varepsilon \sim h$ : We next offer a sketch, in Figure 11.1, of the case $\varepsilon=5^{-3}$ when, by Table 11.1, all maximal errors occur at the first meshpoint in the respective meshes.

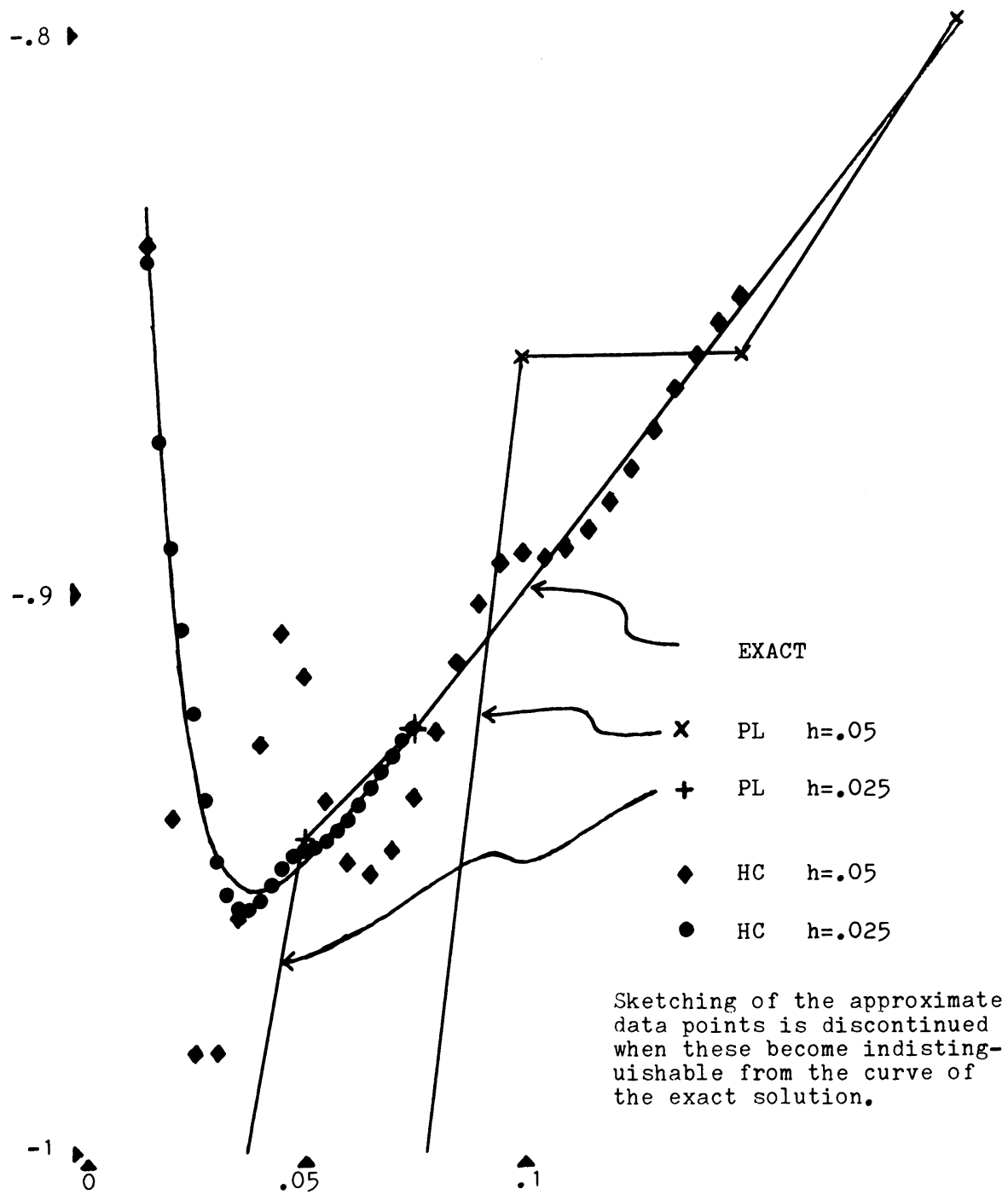

FIGURE 11.1

The transition region when $h \geqslant \varepsilon \simeq h / \ln (1 / h)$ 
Note now that for $h=.025, h / \ln 1 / h=.0068 \leqslant \varepsilon=.008 \leqslant h$. Thus, the situation sketched in Figure 11.1 essentially takes place in a shady region in Figure 10.1. For $d(x) \geqslant c^{\prime} h$ our local estimate (1.8) may be applied (in its one-dimensional version (8.3)) to give

$$
|e(x)| \leqslant C \min _{\chi}\|u-\chi\|_{L_{\infty}[x-d / 2, x+d / 2]}+C \frac{e^{-c_{3} d / h}}{\sqrt{d / h}}
$$

(Here we used Theorem A.2 of Appendix 1 to derive the slightly sharper form of the last term.) Of course, Figure 11.1 looks, en gros, to be governed by local approximability and a decaying term depending only on $d / h$, but it is hard to say which term rules at a specific point. Also, there are two choices for estimating the first term on the right of (11.2): with $\gamma=2$ or 4 we have

$$
\|u-\chi\|_{L_{\infty[\ldots]}} \leqslant \begin{cases}C_{1}\left(\frac{h}{\varepsilon}\right)^{\gamma} e^{-c d / h} & (\chi=\text { interpolant of } u), \\ C_{2} e^{-c d / h} & (\chi=\text { linear part })\end{cases}
$$

and the relative sizes of $C_{1}$ and $C_{2}$ become important in this range.

For $d(x) \geqslant c_{2} h \ln 1 / 2\left(=c_{2} \times 0.15\right)$, the estimate (10.5) takes over and predicts that the influence of the fast decaying term is essentially over. In our case, we happen to know that both terms in (11.2) are fast decaying, but in general this would not be true.

At any rate, even to come this far we have used more specific information about $u$ than we are likely to rigorously secure in a practical two-dimensional problem. Thus, our conclusion is that while the local error estimate (1.8) gives some qualitative insight in the behavior of the approximation close to the boundary layer when $h / \ln 1 / h \lesssim \varepsilon \lesssim h$, Figure 10.1 does well to leave that range as a shady area.

$\varepsilon \ll h$ : When $\varepsilon$ moves well into the range $\varepsilon \leqslant h / \ln 1 / h$, we expect from (the one-dimensional, and also the Hermite cubic analogue of) (10.7) that the error should behave as, for $d \geqslant c_{2} h$,

$$
|e(x)| \leqslant C\left(h^{\gamma}+e^{-c_{3} d / h}\right), \quad \gamma=2 \text { or } 4 .
$$

In our present case, perfect information and Appendix 1 again permit a sharper estimate,

$$
|e(x)| \leqslant C\left(e^{-c d / \varepsilon}+\frac{e^{-c d / h}}{\sqrt{d / h}}\right) \leqslant C \frac{e^{-c d / h}}{\sqrt{d / h}}
$$

In other words, the error at meshpoints should depend only on the meshpoint number, not on $\varepsilon$ or $h$ (for $\varepsilon \leqslant c_{1} h / \ln 1 / h, d(x) \geqslant c_{2} h$ ).

The numerical solution bears out this prediction, as is seen in great detail in Table 11.3. 
TABLE 11.3

The exponentially decreasing pollution in its purest form

\begin{tabular}{|c|c|c|c|c|c|c|c|c|c|}
\hline \multicolumn{3}{|c|}{ Meshpoint \# } & 1 & 2 & 3 & 4 & 9 & 14 & 19 \\
\hline$\varepsilon$ & $\stackrel{\circ}{S}_{h}$ & $h$ & \multirow[b]{2}{*}{.27} & \multirow{3}{*}{$\begin{array}{l}-.70-1 \\
-.66-1\end{array}$} & \multirow{3}{*}{$\begin{array}{l}.19-1 \\
.17-1\end{array}$} & \multirow{3}{*}{$\begin{array}{l}-.49-2 \\
-.43-2\end{array}$} & \multirow{3}{*}{$\begin{array}{l}.65-5 \\
.49-5\end{array}$} & \multirow{3}{*}{$\begin{array}{l}-.85-8 \\
-.54-8\end{array}$} & \multirow{3}{*}{$\begin{array}{l}.10-10 \\
.60-11\end{array}$} \\
\hline $5^{-4}$ & PL & .05 & & & & & & & \\
\hline & & .025 & .25 & & & & & & \\
\hline \multirow[t]{2}{*}{$5^{-5}$} & PL & .05 & .27 & $-.72-1$ & $.19-1$ & $-.51-2$ & $.71-5$ & $-.98-8$ & $.13-10$ \\
\hline & & .025 & .27 & $-.72-1$ & $.19-1$ & $-.51-2$ & $.70-5$ & $-.96-8$ & $.13-10$ \\
\hline \multirow[t]{2}{*}{$5^{-6}$} & PL & .05 & .27 & $-.72-1$ & $.19-1$ & $-.52-2$ & $.71-5$ & $-.98-8$ & $.13-10$ \\
\hline & & .025 & .27 & $-.72-1$ & $.19-1$ & $-.52-2$ & $.71-5$ & $-.98-8$ & $.13-10$ \\
\hline \multirow[t]{2}{*}{$5^{-4}$} & $\mathrm{HC}$ & .05 & $.78-1$ & $.19-1$ & $.53-2$ & $.15-2$ & $.25-5$ & $.42-8$ & $.66-11$ \\
\hline & & .025 & $.68-1$ & $.16-1$ & $.43-2$ & $.11-2$ & $.16-5$ & $.22-8$ & $.30-11$ \\
\hline \multirow[t]{2}{*}{$5^{-5}$} & $\mathrm{HC}$ & .05 & $.82-1$ & $.20-1$ & $.57-2$ & $.16-2$ & $.30-5$ & $.53-8$ & $.89-11$ \\
\hline & & .025 & $82-1$ & $.20-1$ & $.56-2$ & $.16-2$ & $.29-5$ & $.52-8$ & $.94-11$ \\
\hline \multirow[t]{2}{*}{$5^{-6}$} & $\mathrm{HC}$ & .05 & $.82-1$ & $.20-1$ & $.57-2$ & $.16-2$ & $.30-5$ & $.54-8$ & $.91-11$ \\
\hline & & .025 & $.82-1$ & $.20-1$ & $.57-2$ & $.16-2$ & $.30-5$ & $.54-8$ & $.98-11$ \\
\hline
\end{tabular}

Example 11.2. We illustrate our assertation that, away from the boundary layer, local approximability governs asymptotically, in a somewhat less trivial example than the previous. For this purpose, let

$$
u(x)=e^{-x / \varepsilon}+e^{x}-x\left(e+e^{-1 / \varepsilon}\right)-2(1-x),
$$

so that $u$ solves the equation

$$
-\varepsilon^{2} u^{\prime \prime}+u=\left(-\varepsilon^{2}+1\right) e^{x}-x\left(e+e^{-1 / \varepsilon}\right)-2(1-x) .
$$

We take $\varepsilon=5^{-3}$ and work with Hermite cubics. The analogues of (10.5), (10.8) predict

$$
|e(x)|=O\left(h^{4}\right) \quad \text { for } d(x) \geqslant \text { const } \max (h \ln 1 / h, \varepsilon \ln 1 / \varepsilon) .
$$

In Table 11.4 we present the errors for $x=.25, .5$ and .75 , and also the global error, for a range of $h$ 's, and calculate rates of convergence.

\begin{tabular}{|c|c|c|c|c|c|c|c|c|}
\hline \multirow[b]{2}{*}{$h$} & \multicolumn{2}{|c|}{$x=.25$} & \multicolumn{2}{|c|}{$x=.5$} & \multicolumn{2}{|c|}{$x=.75$} & \multicolumn{2}{|c|}{ GLOBAL } \\
\hline & Error & Rate & Error & Rate & Error & Rate & Error & Rate \\
\hline $20^{-1}$ & $.775-4$ & & $.606-7$ & & $.184-7$ & & $.33-1$ & \\
\hline $40^{-1}$ & $.453-8$ & 14 & $.895-9$ & 6.1 & $.118-8$ & 4.0 & $.94-2$ & 1.8 \\
\hline $80^{-1}$ & $.435-10$ & 6.7 & $.559-10$ & 4.0 & $.718-10$ & 4.0 & $.16-2$ & 2.6 \\
\hline $160^{-1}$ & $.272-11$ & 4.0 & $.349-11$ & 4.0 & $.449-11$ & 4.0 & $.19-3$ & 3.1 \\
\hline
\end{tabular}

TABLE 11.4

Calculated rates of convergence

We see that the expected local rate $h^{4}$ eventually appears. If one calculates $e^{-d / h} / \sqrt{d / h}$ one sees that, due to the miniscule errors, it probably influences the errors for $x=.25, h=20^{-1}, 40^{-1}, 80^{-1}$ and for $x=.5, h=20^{-1}, 40^{-1}$. This is the explanation why the asymptotic range appears later in the left part of the table. We also remark that the global error always occurred at the first meshpoint, as expected. 
Example 11.3. We give a two-dimensional example of the pollution effect in its purest form.

We solved the problem, $\varepsilon=.001$,

$$
\begin{gathered}
-\varepsilon^{2} \Delta u+u=0, \quad 0 \leqslant x, y \leqslant 1, \\
u(x, y)=e^{-x / \varepsilon}+e^{-y / \varepsilon} \quad \text { on the boundary, }
\end{gathered}
$$

which has the obvious exact solution. We used triangular linear elements. The mesh was essentially the product of a uniform subdivision in the $y$-direction with $h=.05$, and a subdivision $\left\{x_{j+1}\right\}_{0}^{20}$ in the $x$-direction, $x_{j+1}=.01 j+.002 j^{2}$. Thus, the $x$-mesh was slightly refined towards the origin. There were 361 interior nodes and 800 elements.

In Figure 11.2 we display the errors at meshpoints in a subregion of the unit square. We leave it to the reader to analyze it along the lines of Example 11.1; the picture is clear.
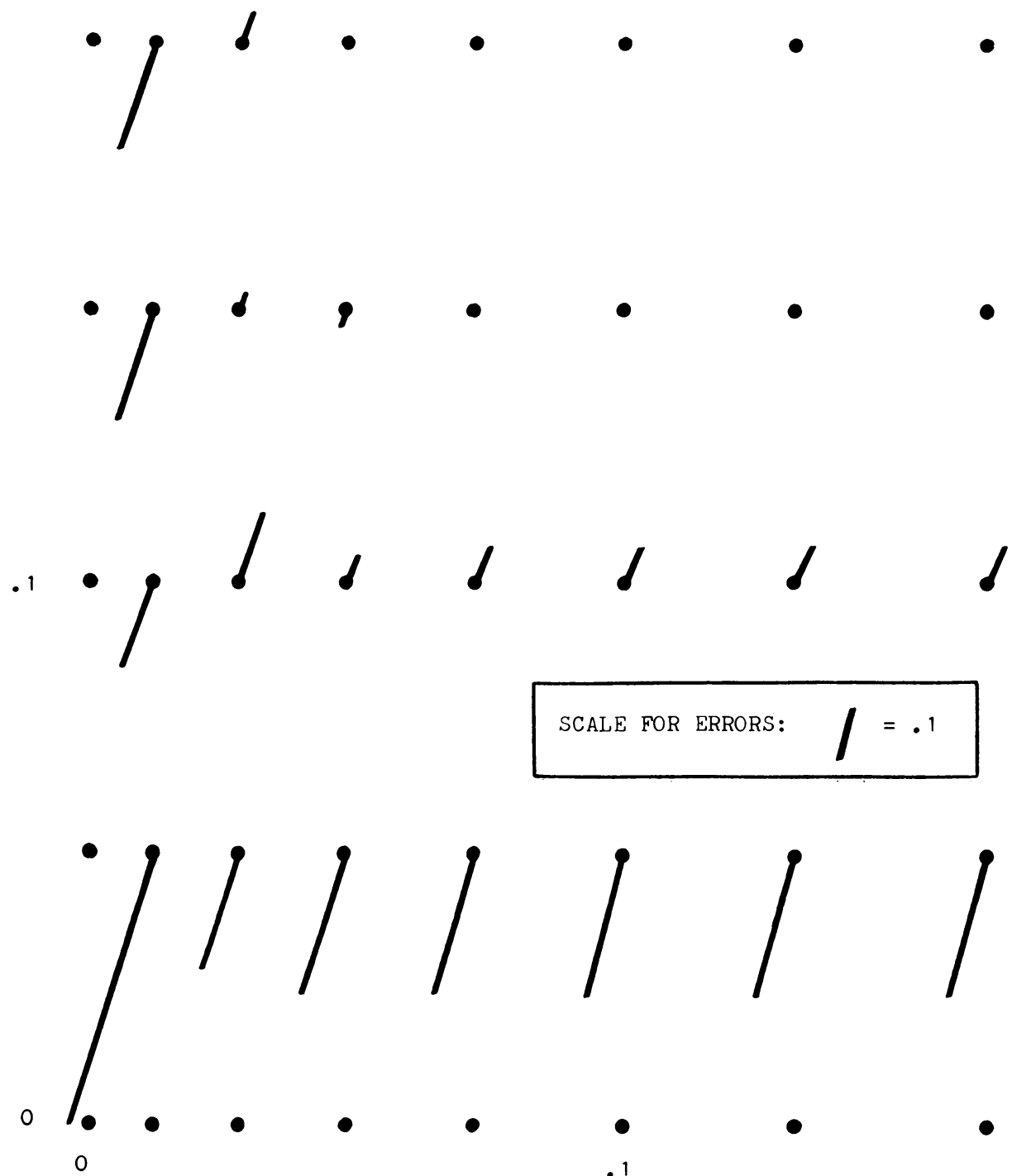

FIGURE 11.2

A two-dimensional example of the pollution effect in its pure form 
Example 11.4. This example shows that, when $a(x)$ vanishes, the exponential decrease of pollution is lost.

Consider the transmission problem

$$
\begin{gathered}
-\varepsilon^{2} u^{\prime \prime}+\sigma(x) u=\sigma(x)(3 x-1), \quad 0 \leqslant x \leqslant 2, \\
u(0)=u(2)=0,
\end{gathered}
$$

where

$$
\sigma(x)= \begin{cases}0, & 0 \leqslant x \leqslant 1 \\ 1, & 1 \leqslant x \leqslant 2\end{cases}
$$

With $E=e^{-1 / \varepsilon}$, the exact solution is

$$
u(x)=\left\{\begin{array}{r}
\left(\frac{-1-E^{2}+10 E}{\varepsilon+1-E^{2}(\varepsilon-1)}+3\right) x, \quad 0 \leqslant x \leqslant 1, \\
(\varepsilon+5 E(1-\varepsilon)) e^{-(x-1) / \varepsilon}+(5+5 \varepsilon-E \varepsilon) e^{-(2-x) / \varepsilon}+3 x-1, \\
1 \leqslant x \leqslant 2 .
\end{array}\right.
$$

We choose $\varepsilon=8.10^{-6}$.

Thus, on $[0,1]$, the solution is merely a linear function; on $[1,2]$, near 1 there is a boundary layer of the usual kind but weak in magnitude whereas at 2 , there is a stronger boundary layer.

On $[1,2]$, where $\sigma(x)=1$, our theory suggests exponential decay in error as $e^{-\operatorname{dist}(x, p) / h}$ away from $p=1,2$, respectively. On $[0,1]$, where $\sigma(x)=0$, our present theory does not apply. However, local error estimates for nonperturbed Dirichlet problems, Schatz-Wahlbin [24], say that

$$
\begin{aligned}
\left|\left(u-u_{h}\right)(x)\right| \leqslant C \min _{\chi \in \delta_{h}}\|u-\chi\|_{L_{\infty}\left(I_{d}\right)}+C d^{-1 / 2}\left\|u-u_{h}\right\|_{L_{2}\left(I_{d}\right)} \\
\simeq C d^{-1 / 2}\left\|u-u_{h}\right\|_{L_{2}\left(I_{d}\right)}
\end{aligned}
$$

and so predict no exponential decay in error.

\begin{tabular}{|c|c|c|c|c|c|c|c|c|c|c|c|}
\hline mesh pt. & 0 & 2 & 4 & 6 & 8 & 10 & 12 & 14 & 16 & 18 & \\
\hline \multirow[t]{3}{*}{ error } & 0 & $.6-3$ & $.1-3$ & $.2-2$ & $.2-2$ & $.3-2$ & $.4-2$ & $.4-2$ & $.5-2$ & $.5-2$ & \\
\hline & 20 & 22 & 24 & 26 & 28 & 30 & 32 & 34 & 36 & 38 & 40 \\
\hline & $.8-5$ & $.2-8$ & $.2-8$ & $.3-7$ & $.3-6$ & $.4-5$ & $.5-4$ & $.7-3$ & $.8-2$ & .1 & 0 \\
\hline
\end{tabular}

Computing with Hermite cubics for $h=40^{-1}$ (and hence a meshpoint placed at $x=1$ ), the following errors ensued, reported here at every second meshpoint, Table 11.5.

\section{TABLE 11.5}

Damping of pollution depending on the positivity of the coefficient a $(x)$

The decrease in error away from $x=2$ (meshpoint 40 ) is easily discerned; to the right of $x=1$ (meshpoint 20) the exponential decay is less noticeable, since the boundary layer is very weak here. However, on $[0,1]$, in spite of the fact that the solution here is perfectly approximable by Hermite cubics, pollution is rampant all through and does not decay. 
The same pattern occurs if $x=1$ is placed inside a mesh interval, the errors then being magnified by roughly a factor 5 on $[0,1]$, but staying the same on $[1,2]$ except near 1 , as expected.

Example 11.5. Pollution absorbent mesh. One may draw a limited practical conclusion from our analysis. Say that we have a problem on $[0,1]$ and desire "good" accuracy on [.1,.9] uniformly in $\varepsilon$, with a fixed mesh. The nature of the local error, in particular the pollution term which only depends on the number of meshpoints away from the boundary for low $\varepsilon$, suggests putting a few extra meshpoints inside $[0, .1]$ and $[.9,1]$, for the purpose of absorbing the pollution. Thus the aim is not actually to resolve the boundary layer, which would demand a mesh refinement depending on $\varepsilon$ (and would possibly be bothersome in many two-dimensional codes if a large number of $\varepsilon$ 's are to be investigated).

Observe that, from the asymptotic point of view as $h \rightarrow 0$, since $[.1,9]$ is a fixed interior subinterval, there would be no problem of high accuracy if the solution is smooth. We are having in mind moderate to large mesh sizes here.

We illustrate the principle in the problem of Example 11.2 (and we use our a priori knowledge that a boundary layer occurs only at the left). Taking a mesh with 10 subintervals, the problem was run for a range of $\varepsilon$ 's, and with two different meshes. First, a uniform mesh, and secondly, a mesh with 4 pieces of length .025 in $[0, .1]$ and 6 parts of length .15 in $[.1,1]$.

The results are given in Table 11.6; the error was calculated only at meshpoints.

TABLE 11.6

Uniform mesh vs. pollution absorbent mesh

\begin{tabular}{l|c|c|}
\cline { 2 - 3 } & \multicolumn{2}{|c|}{$\|e\|_{L_{\infty}[1,1]}$} \\
\cline { 2 - 3 }$\varepsilon$ & Uniform Mesh & Pollution Absorbent Mesh \\
\cline { 2 - 3 } $5^{0}$ & $.30-6$ & $.16-5$ \\
$5^{-1}$ & $.42-4$ & $.11-3$ \\
$5^{-2}$ & $.55-2$ & $.12-2$ \\
$5^{-3}$ & $.62-1$ & $.90-4$ \\
$5^{-4}$ & $.81-1$ & $.10-2$ \\
$5^{-5}$ & $.82-1$ & $.14-2$ \\
$5^{-6}$ & $.82-1$ & $.15-2$ \\
\end{tabular}

We conclude that the pollution absorbing idea works. For the error uniformly in $\varepsilon$ over [.1,1], $\sup _{0<\varepsilon \leqslant 1}\|e\|_{L_{\infty}[.1,1]}$, we have obtained a 50 -fold reduction over the uniform mesh.

Example 11.6. Comparison with some specially designed finite difference schemes. In the one-dimensional case, Miller [17], Niijima [19], [20] and Shiskin and Titov [26] have investigated special three-point finite difference schemes which converge uniformly in $\varepsilon$ with respect to the maximum norm over meshpoints but which do not attempt to resolve the boundary layer for small $\varepsilon$. The construction in [17] and [26], where the same scheme is analyzed, is based on exponential fitting, whereas the two schemes in [19] and [20] are motivated by Liouville-Green transformations. We refer the reader to the papers mentioned for details. 
In [20] the three schemes were compared when applied to the problem $(t=$ $2 x /(x+1))$

$$
\begin{gathered}
-\varepsilon^{2} u^{\prime \prime}+\frac{4}{(x+1)^{4}}(1+\varepsilon(x+1)) u \\
=\frac{-4}{(x+1)^{4}}\left[\left(1+\varepsilon(x+1)+4 \pi^{2} \varepsilon^{2}\right) \cos (2 \pi t)\right. \\
\left.-2 \pi \varepsilon^{2}(x+1) \sin (2 \pi t)+\frac{3(1+\varepsilon(x+1)) e^{-1 / \varepsilon}}{1-e^{-1 / \varepsilon}}\right], \quad 0 \leqslant x \leqslant 1, \\
u(0)=2, \quad u(1)=-1 .
\end{gathered}
$$

This problem has the exact solution

$$
u(x)=-\cos (2 \pi t)+\frac{3\left(e^{-t / \varepsilon}-e^{-1 / \varepsilon}\right)}{1-e^{-1 / \varepsilon}} .
$$

We shall reproduce, in Table 11.7 , the results for the uniform step $1 / 32$ in the finite difference schemes. In the table we adjoin the results for the Galerkin method with Hermite cubics, applied with a pollution absorbent mesh (on the left only) constructed as follows. With $H=1 / 32$, the interval $[0, H]$ was divided into four uniform subintervals, to absorb pollution, and the rest, $[H, 1]$, was partitioned into twelve equal pieces. Thus, the number of unknowns in each problem was similar. The maximal error was taken over each meshpoint in the three difference methods and, for the Galerkin method, over the meshpoints in $[H, 1]$ (including $x=H$ ).

Note that we are comparing the general purpose Galerkin method to highly specialized numerical methods, available only in one-dimensional smooth cases.

TABLE 11.7

Comparison with some special difference schemes

\begin{tabular}{l|c|l|l|l|}
\cline { 2 - 5 }$\varepsilon$ & Miller [17] & Niijima [19] & Niijima [20] & Galerkin HC \\
\cline { 2 - 5 } $10^{-1}$ & $.65-2$ & $.48-2$ & $.62-4$ & $.34-2$ \\
$10^{-1.5}$ & $.71-2$ & $.77-2$ & $.26-4$ & $.11-1$ \\
$10^{-2}$ & $.26-2$ & $.66-2$ & $.28-4$ & $.80-3$ \\
$10^{-2.5}$ & $.40-3$ & $.28-2$ & $.27-4$ & $.38-3$ \\
$10^{-3}$ & $.41-4$ & $.97-3$ & $.86-5$ & $.22-2$ \\
$10^{-3.5}$ & $.39-5$ & $.31-3$ & $.32-5$ & $.30-2$ \\
$10^{-4}$ & $.66-6$ & $.10-3$ & $.30-5$ & $.31-2$ \\
\hline
\end{tabular}

The large errors for rather moderate $\varepsilon$ are due to the very abrupt change in mesh size.

For further comparison it might be remarked that, for a uniform step of length $1 / 16$, the quantity $\max _{\varepsilon} \max _{j}|e(j h)|$ was $.25-1, .21-1$ and $.20-3$ in the difference schemes [17], [19] and [20], respectively.

We leave it to the interested reader to investigate how methods involving matched asymptotic expansions (and combinations of such with finite element methods) compare; this is easy to do in most examples above. 
12. A Nonlinear Problem. We show how our results apply to a nonlinear problem. Let $u$ solve

$$
\begin{array}{ll}
-\varepsilon^{2} \Delta u+b(x, u ; \varepsilon)=f(x ; \varepsilon) & \text { in } \Re, \\
u=0 & \text { on } \partial \Re,
\end{array}
$$

where $b(x, 0 ; \varepsilon) \equiv 0, b$ is absolutely continuous in $u$, and with some constant $a_{0}>0$,

$$
\frac{\partial b}{\partial u}(x, u ; \varepsilon) \geqslant a_{0}>0 \quad \text { for } x \in \bar{R}, 0<\varepsilon \leqslant 1, u \in R^{1} .
$$

Further assume that $\partial b / \partial u$ is bounded above on compact $u$-intervals.

The result of Lemma A.l of the Appendix extends to this situation and thus

$$
\|u\|_{L_{\infty}} \leqslant \frac{1}{a_{0}}\|f\|_{L_{\infty}} .
$$

The existence of a unique solution to (12.1) can be obtained by monotonicity methods, see Brezis [5] or Lions [15].

We thus have an easily computable bound for $|u|$ that is independent of $\varepsilon$. We assume in general that we know bounds $u_{0}$ and $u_{1}$ such that

$$
u_{0} \leqslant u(x ; \varepsilon) \leqslant u_{1} \text { for } x \in \bar{R}, 0<\varepsilon \leqslant 1 .
$$

To find an approximation $u_{h}$ by the finite element method we first change $b(x, u ; \varepsilon)$ to $\tilde{b}(x, u ; \varepsilon)$ where

$$
\tilde{b}(u)= \begin{cases}b\left(u_{0}\right)+\frac{\partial b}{\partial u}\left(u_{0}\right)\left(u-u_{0}\right) & \text { for } u \leqslant u_{0}, \\ b(u) & \text { for } u_{0} \leqslant u \leqslant u_{1}, \\ b\left(u_{1}\right)+\frac{\partial b}{\partial u}\left(u_{1}\right)\left(u-u_{1}\right), & \text { for } u \geqslant u_{1} .\end{cases}
$$

This modification is necessary in order to apply our theory when $\partial b / \partial u$ may grow with $|u|$, at least in an effortless way. It is probably not necessary in practical computations but it is very easy to incorporate if desired.

We define the approximate solution $u_{h} \in \mathcal{S}_{h}$ by

$$
\varepsilon^{2}\left(\nabla u_{h}, \nabla \chi\right)+\left(\tilde{b}\left(u_{h}\right), \chi\right)=(f, \chi) \text { for } \chi \in \mathcal{S}_{h} .
$$

The existence of $u_{h}$ is again guaranteed by monotonicity methods.

In the range given in (12.3), $b$ and $\tilde{b}$ coincide and thus $u-u_{h}$ satisfies

$$
\varepsilon^{2}\left(\nabla\left(u-u_{h}\right), \nabla \chi\right)+\left(a\left(u-u_{h}\right), \chi\right)=0 \text { for } \chi \in \mathcal{S}_{h},
$$

where

$$
a(x ; \varepsilon)=\int_{0}^{1} \frac{\partial \tilde{b}}{\partial u}\left(t u(x)+(1-t) u_{h}(x)\right) d t
$$

With $a_{1}=\sup \left\{\partial b(x, u ; \varepsilon) / \partial u: u_{0} \leqslant u \leqslant u_{1}, 0<\varepsilon \leqslant 1, x \in \bar{R}\right\}$ and $a_{0}$ as in (12.2) we have now

$$
0<a_{0} \leqslant a(x ; \varepsilon) \leqslant a_{1} .
$$


From (12.5) and (12.6) we see that the global and local error estimates of Theorems 6.1 and 7.1 immediately apply. We can thus state the following

Theorem 12.1. Assume (1.2), (12.2), (12.3) and A.1-A.6 of Section 3. Let $u$ be a continuous function vanishing on $\partial \Re$ and solving (12.1) in the weak sense, and let $u_{h} \in \mathcal{S}_{h}$ be given by (12.4).

There exists a constant $C$, independent of $u, u_{h}, \varepsilon$ and $h$ but depending on $u_{0}, u_{1}$ (through $a_{0}, a_{1}$ ) such that

$$
\left\|u-u_{h}\right\|_{L_{\infty}(\Re)} \leqslant \ln \left(C+\frac{\varepsilon}{h}\right) \min _{\chi \in \mathcal{S}_{h}}\|u-\chi\|_{L_{\infty}(\Re)} .
$$

Further, let $x_{0} \in \bar{\Re}$, let $D$ be a disc of radius $d$ around $x_{0}$ and set $\Omega_{d}=D \cap \Re$. There exist positive constants $c_{1}, c_{2}$ and $C$, independent of $u, u_{h}, \varepsilon, h, x_{0}$ and $d$ such that for $d \geqslant c_{1} h$,

$$
\begin{aligned}
& \left|\left(u-u_{h}\right)\left(x_{0}\right)\right| \\
& \quad \leqslant C \ln ^{1 / 2}\left(\frac{d}{h}\right)\left\{\ln \left(C+\frac{\varepsilon}{h}\right) \min _{\chi \in \delta_{h}}\|u-\chi\|_{L_{\infty}\left(\Omega_{d}\right)}+\frac{1}{d} e^{-c_{2} d /(\varepsilon+h)}\left\|u-u_{h}\right\|_{L_{2}\left(\Omega_{d}\right)}\right\} .
\end{aligned}
$$

Appendix 1. Some Global $L_{2}$-Estimates in the Dirichlet Problem. We consider the problem

$$
\begin{cases}-\varepsilon^{2} \Delta u+a u=f & \text { in } R \subset \subset R^{2}, \\ u=0 & \text { on } \partial \mathscr{R}\end{cases}
$$

under the general basic assumptions (1.2) and (1.4). The finite element spaces are subject to the conditions of Section 3. We remark that it is easier to treat the Neumann problem in an analogous way, and we leave that case to the reader.

We shall derive estimates for $\left\|u-u_{h}\right\|_{L_{2}}$ under weak assumptions on $f$ and $a$. Our main result is Theorem A.2. Due to the form of the boundary layer and our wish to allow data with jump discontinuities, a certain interpolation space between $H^{1}$ and $L_{2}$ turns out to be useful. We proceed to define and discuss this space.

For $0<\theta<1$, let $H^{\theta, \infty}=\left[\stackrel{\circ}{H}^{1}, L_{2}\right]_{\theta, \infty}$ denote the space given by the norm

$$
\|v\|_{\theta, \infty}=\sup _{t>0} \frac{K(t, v)}{t^{\theta}}
$$

where

$$
K(t, v)=\inf _{\substack{v=v_{0}+v_{1} \\ v_{0} \in L_{2}, v_{1} \in \dot{H}^{1}}}\left\|v_{0}\right\|_{L_{2}}+t\left\|v_{1}\right\|_{\dot{H}^{1}} .
$$

We refer to Bergh and Löfström [2, Chapter 3] for more information about these concepts.

Of particular interest to us is the space $H^{1 / 2, \infty}$. Even for smooth data in (A.1) but with $\left.f\right|_{\partial \Re} \neq 0$, the boundary layer precludes $u$ from being in $H^{\theta, \infty}$ uniformly in $\varepsilon$, for $\theta>1 / 2$ (we shall not prove this). Thus, as it will turn out, $H^{1 / 2, \infty}$ is a good choice. Also, $H^{1 / 2, \infty}$ is a reasonable space to measure $f$ in: For, $f$ will then not be subject to boundary conditions, and $f$ is allowed to have jumps in $R$. In fact, pondering the reduced equation $u=f / a$, taking $f$ in $H^{1 / 2, \infty}$ seems to nicely tie in with the 
roughness from the boundary layer; it will also allow jumps in the coefficient $a$, while still $u \in H^{1 / 2, \infty}$, as we shall see.

To somewhat elucidate these spaces we state and prove the following simple result which shows that $H^{1 / 2, \infty}$ does not demand boundary conditions. (This is in contrast to the Hilbertian interpolation space $\left[\stackrel{\circ}{H}^{1}, L_{2}\right]_{1 / 2,2}=H_{00}^{1 / 2}$, cf., e.g., Lions and Magenes [16, Chapter 1, Section 11, Theorem 11.7].)

Proposition A.1 (Lions [14, Chapter II, Section 5, Lemma 5.1]).

$$
H^{1}(\Re) \subseteq H^{1 / 2, \infty}(\Re)
$$

with continuous inclusion.

Proof. By use of local charts, it suffices to consider the case of $\Re=\left\{x=\left(x_{1}, x_{2}\right)\right.$ : $\left.x_{2}>0\right\}$. For $v \in H^{1}$ and $t>0$, set

$$
v_{0 t}(x)=v\left(x_{1}, \frac{x_{2}}{t}\right), \quad v_{1 t}(x)=v(x)-v_{0 t}(x) .
$$

Then

$$
\left\|v_{0 t}\right\|_{L_{2}} \leqslant t^{1 / 2}\|v\|_{L_{2}}
$$

whereas $v_{1 t} \in \stackrel{H}{ }^{1}$ with

$$
\left\|v_{1 t}\right\|_{H^{1}} \leqslant t^{-1 / 2}\|v\|_{H^{1}}
$$

It follows that $K(t, v) \leqslant C t^{1 / 2}\|v\|_{H^{1}}$ and so $v \in H^{1 / 2, \infty}$.

In a similar way, if $\Gamma$ is a Lipschitz curve partitioning $R$ into $\Re_{1}$ and $\Re_{2}$, and $v$ is smooth on $\bar{\Re}_{1}$ and $\bar{\Re}_{2}$, then $v \in H^{1 / 2, \infty}$. To see this, smooth $v$ across $\Gamma$ by a mollifier, cf. (A.3)-(A.6) below.

We next show two simple lemmas.

Lemma A.1.

$$
\|u\|_{L_{\infty}} \leqslant \frac{1}{a_{0}}\|f\|_{L_{\infty}} .
$$

Proof. In case $u \in \mathcal{C}^{2}(\Re)$ the assertation is immediate by considering the sign of $\Delta u$ at interior positive maxima or negative minima.

In general we proceed as follows: Multiply the equation (4.1) by $u^{p-1}, p$ even, and integrate by parts to arrive at

$$
\varepsilon^{2} \int|\nabla u|^{2}(p-1) u^{p-2}+\int a u^{p}=\int f u^{p-1} .
$$

Hölder's inequality then gives

$$
a_{0}\|u\|_{L_{p}}^{p} \leqslant\|f\|_{L_{p}}\|u\|_{L_{p}}^{p-1}
$$

and the lemma follows upon letting $p$ tend to infinity.

LeMmA A.2. If, in addition to (1.2) and (1.4), $\nabla a \in L_{\infty}$ and $f \in \stackrel{\circ}{H}^{1}$, then

$$
\varepsilon\|u\|_{H^{2}}+\|u\|_{H^{1}} \leqslant C\left(\|f\|_{H^{1}}+\|(\nabla a) u\|_{L_{2}}\right) .
$$

Proof. Multiply the equation (A.1) by $\Delta u$ and integrate by parts to obtain

$$
\varepsilon^{2}\|\Delta u\|^{2}+\|\sqrt{a} \nabla u\|^{2}=\int \nabla f \cdot \nabla u-\int(\nabla a) u \nabla u .
$$


Thus,

$$
\varepsilon\|\Delta u\|+\|\nabla u\| \leqslant C(\|\nabla f\|+\|\nabla a u\|)
$$

and, since $\|u\|_{H^{2}} \leqslant C\|\Delta u\|$ for the domains under consideration, the lemma follows.

COROllary. If $\|\nabla a\|_{L_{\infty}} \leqslant C$ and $f \in \stackrel{\circ}{H}^{1}$, then

$$
\varepsilon\|u\|_{H^{2}}+\|u\|_{H^{1}} \leqslant C\|f\|_{H^{1}} \text {. }
$$

We are now ready to state a first simple $L_{2}$ error estimate. The general assumptions are (1.2) and (1.4) and those of Section 3.

TheOrem A.1. (i) For $f \in L_{2}$,

$$
\left\|u-u_{h}\right\|_{L_{2}} \leqslant \begin{cases}C\|f\|_{L_{2}}, & 0<\varepsilon \leqslant h, \\ \frac{C h^{2}}{\varepsilon^{2}}\|f\|_{L_{2}}, & h \leqslant \varepsilon \leqslant 1 .\end{cases}
$$

(ii) If $\|\nabla a\|_{L_{\infty}} \leqslant C$ and $f \in H^{1 / 2, \infty}$, then

$$
\left\|u-u_{h}\right\|_{L_{2}} \leqslant \begin{cases}C \sqrt{h}\|f\|_{1 / 2, \infty}, & 0<\varepsilon \leqslant h, \\ \frac{C h^{2}}{\varepsilon^{3 / 2}}\|f\|_{1 / 2, \infty}, & h \leqslant \varepsilon \leqslant 1 .\end{cases}
$$

(iii) If $\|\nabla a\|_{L_{\infty}} \leqslant C$ and $f \in \stackrel{\circ}{H}^{1}$, then

$$
\left\|u-u_{h}\right\|_{L_{2}} \leqslant \begin{cases}C h\|f\|_{H^{\circ}}, & 0<\varepsilon \leqslant h, \\ \frac{C h^{2}}{\varepsilon}\|f\|_{H^{\circ}}, & h \leqslant \varepsilon \leqslant 1 .\end{cases}
$$

Proof. The first estimate in (i) is trivial; the second follows from Lemma 4.1 and Lemma 2.1.

For (iii), use Lemma 4.1 and then the Corollary to Lemma A.2.

The estimates in (ii) now follow by interpolation. For the reader unfamiliar with interpolation arguments involving the $K$-functional, we give the simple reasoning, in the case $0<\varepsilon \leqslant h$. Write $f=f_{0}+f_{1}$ where $f_{0} \in L_{2}, f_{1} \in \stackrel{\circ}{H}^{1}$. Then $u=u_{0}+u_{1}$ where $u_{i}$ solve Dirichlet problems with right-hand sides $f_{i}$. By linearity, using (i) and (iii),

$$
\left\|u-u_{h}\right\| \leqslant\left\|u_{0}-P_{h}^{\varepsilon} u_{0}\right\|+\left\|u_{1}-P_{h}^{\varepsilon} u_{1}\right\| \leqslant C\left\|f_{0}\right\|_{L_{2}}+C h\left\|f_{1}\right\|_{H^{1}} .
$$

Considering all possible $f_{0}$ and $f_{1}$, we see that $\left\|u-u_{h}\right\| \leqslant C K(h, f)$ and since $K(h, f) \leqslant \sqrt{h}\|f\|_{1 / 2, \infty}$ we are done.

Remark A.1. By the same techniques it follows that, if $\|\nabla a\|_{L_{\infty}} \leqslant C$,

$$
\|u\|_{1 / 2, \infty} \leqslant C\|f\|_{1 / 2, \infty} .
$$

Finally we want to consider the case when $a$ is not smooth, so that $\nabla a$ does not exist, and prove an analogue of Theorem A.1(ii) in this situation. The assumptions on $a$ and $f$ will be such as to cover many practical situations. Thus let $a \in H^{1 / 2, \infty} \cap$ $L_{\infty}$, where we now may take $H^{1 / 2, \infty}=\left[H^{1}, L_{2}\right]_{1 / 2, \infty}$ (which might be technically simpler for checking). Considering then the reduced equation $u \cong f / a$ for small $\varepsilon$, it 
is unlikely that $u$ is smoother than in $H^{1 / 2, \infty}$ uniformly in $\varepsilon$, and hence no better rate of convergence than $\sqrt{h}$ can be expected for small $\varepsilon$. Thus, the estimate of case (ii) of Theorem A.l ought to be the best result possible, and we shall (almost) obtain it.

THeorem A.2. Assume (1.2), (1.4) and the conditions on $\dot{S}_{h}$ in Section 3. Let $a \in H^{1 / 2, \infty} \cap L_{\infty}$. Then

$$
\left\|u-u_{h}\right\|_{L_{2}} \leqslant \begin{cases}C \sqrt{h}\left(\|f\|_{1 / 2, \infty}+\|f\|_{L_{\infty}}\right), & 0<\varepsilon \leqslant h, \\ \frac{C h^{2}}{\varepsilon^{3 / 2}}\left(\|f\|_{1 / 2, \infty}+\|f\|_{L_{\infty}}\right), & h \leqslant \varepsilon \leqslant 1 .\end{cases}
$$

Proof. We shall need to smoothen the coefficient $a$. The class of $a$ 's considered makes it possible to construct $\tilde{a}=\tilde{a}(\delta)$ for $0<\delta \leqslant 1$ such that, with constants uniform in $\delta$,

$$
\begin{aligned}
& 0<\frac{a_{0}}{2} \leqslant \tilde{a} \leqslant 2 a_{1}, \\
& \|a-\tilde{a}\|_{L_{2}} \leqslant C \sqrt{\delta}, \\
& \|\nabla \tilde{a}\|_{L_{2}} \leqslant \frac{C}{\sqrt{\delta}}, \\
& \|\nabla \tilde{a}\|_{L_{\infty}} \leqslant \frac{C}{\delta} .
\end{aligned}
$$

To see this, use a mollifier of standard type.

We shall give the details of the proof in the case $0<\varepsilon \leqslant h$ and indicate the modifications necessary for $h \leqslant \varepsilon \leqslant 1$.

First smooth $a$ to $\tilde{a}=\tilde{a}(h)(\delta=h)$ and define $\tilde{u}$ by

$$
-\varepsilon^{2} \Delta \tilde{u}+\tilde{a} \tilde{u}=f, \quad \tilde{u}=0 \quad \text { on } \partial \Re
$$

then $u-\tilde{u}$ satisfies

(A.8) $-\varepsilon^{2} \Delta(u-\tilde{u})+\tilde{a}(u-\tilde{u})=(\tilde{a}-a) u, \quad u-\tilde{u}=0 \quad$ on $\partial \Re$.

Write now $\left(u_{h}=P_{h}^{\varepsilon} u\right)$

$$
u-u_{h}=\tilde{u}-P_{h}^{\varepsilon} \tilde{u}+(u-\tilde{u})-P_{h}^{\varepsilon}(u-\tilde{u}) .
$$

From (A.8) we find, using also Lemma A.1 and (A.3), (A.4),

$$
\|u-\tilde{u}\| \leqslant C\|(\tilde{a}-a) u\| \leqslant C\|\tilde{a}-a\|_{L_{2}}\|u\|_{L_{\infty}} \leqslant C \sqrt{h}\|f\|_{L_{\infty}}
$$

and similarly

$$
\left\|P_{h}^{\epsilon}(u-\tilde{u})\right\| \leqslant C \sqrt{h}\|f\|_{L_{\infty}} .
$$

By (A.9), (A.10) and (A.11) it remains to estimate $\tilde{u}-P_{h}^{\varepsilon} \tilde{u}$. Let $f=f_{0}+f_{1}$ with $f_{0} \in L_{2}, f_{1} \in \dot{H}^{1}$. Then $\tilde{u}=\tilde{u}_{0}+\tilde{u}_{1}$ where

$$
-\varepsilon^{2} \Delta \tilde{u}_{i}+\tilde{a} \tilde{u}_{i}=f_{i}, \quad \tilde{u}_{i}=0 \quad \text { on } \partial \Re, i=0,1,
$$

and

$$
\tilde{u}-P_{h}^{\varepsilon} \tilde{u}=\left(\tilde{u}_{0}-P_{h}^{\varepsilon} \tilde{u}_{0}\right)+\left(\tilde{u}_{1}-P_{h}^{\varepsilon} \tilde{u}_{1}\right) .
$$


Then

(A.13)

$$
\left\|\tilde{u}_{0}-P_{h}^{\varepsilon} \tilde{u}_{0}\right\| \leqslant\left\|\tilde{u}_{0}\right\|+\left\|P_{h}^{\varepsilon} \tilde{u}_{0}\right\| \leqslant C\left\|f_{0}\right\|
$$

and, using Lemma 4.1 and Lemma A.2,

$$
\left\|\tilde{u}_{1}-P_{h}^{\varepsilon} \tilde{u}_{1}\right\| \leqslant C h\left\|\tilde{u}_{1}\right\|_{H^{1}} \leqslant C h\left(\left\|f_{1}\right\|_{H^{\prime}}+\left\|(\nabla \tilde{a}) \tilde{u}_{1}\right\|\right) .
$$

Here, by (A.5), Lemma A.1 and (A.6),

(A.15) $\left\|(\nabla \tilde{a}) \tilde{u}_{1}\right\| \leqslant\left\|(\nabla \tilde{a}) \tilde{u}_{0}\right\|+\|(\nabla \tilde{a}) \tilde{u}\|$

$$
\leqslant\|\nabla \tilde{a}\|_{L_{\infty}}\left\|\tilde{u}_{0}\right\|_{L_{2}}+\|\nabla \tilde{a}\|_{L_{2}}\|\tilde{u}\|_{L_{\infty}} \leqslant \frac{C}{h}\left\|f_{0}\right\|_{L_{2}}+\frac{C}{\sqrt{h}}\|f\|_{L_{\infty}} .
$$

Inserting (A.14) and (A.15) in (A.13) and considering all $f_{0}, f_{1}$,

$$
\begin{aligned}
\left\|\tilde{u}-P_{h}^{\varepsilon} \tilde{u}\right\| & \leqslant C\left(\left\|f_{0}\right\|+h\left\|f_{1}\right\|_{H^{1}}\right)+C \sqrt{h}\|f\|_{L_{\infty}} \\
& \leqslant C \sqrt{h}\left(\|f\|_{1 / 2, \infty}+\|f\|_{L_{\infty}}\right) .
\end{aligned}
$$

As noted above, this concludes the case $0<\varepsilon \leqslant h$.

In the case $h \leqslant \varepsilon \leqslant 1$, one uses instead $\tilde{a}=\tilde{a}(\varepsilon)(\delta=\varepsilon)$. Then by Lemma 4.1, Lemma 2.1 and arguments similar to the ones above, instead of (A.10) and (A.11) we find

$$
\begin{aligned}
\left\|(u-\tilde{u})-P_{h}^{\varepsilon}(u-\tilde{u})\right\| & \leqslant C h^{2}\|u-\tilde{u}\|_{H^{2}} \\
& \leqslant \frac{C h^{2}}{\varepsilon^{2}}\|(\tilde{a}-a) u\|_{L_{2}} \leqslant \frac{C h^{2}}{\varepsilon^{3 / 2}}\|f\|_{L_{\infty}} .
\end{aligned}
$$

The estimates in (A.13) et seq. now run as follows, using in particular Lemma A.2.

$$
\begin{gathered}
\left\|\tilde{u}_{0}-P_{h}^{\varepsilon} \tilde{u}_{0}\right\| \leqslant C h^{2}\left\|\tilde{u}_{0}\right\|_{H^{2}} \leqslant \frac{C h^{2}}{\varepsilon^{2}}\left\|f_{0}\right\|, \\
\left\|\tilde{u}_{1}-P_{h}^{\varepsilon} \tilde{u}_{1}\right\| \leqslant C h^{2}\left\|\tilde{u}_{1}\right\|_{H^{2}} \leqslant \frac{C h^{2}}{\varepsilon}\left(\left\|f_{1}\right\|_{H^{1}}+\left\|\nabla \tilde{a} \tilde{u}_{1}\right\|\right) \\
\leqslant \frac{C h^{2}}{\varepsilon}\left(\left\|f_{1}\right\|_{H^{1}}+\left\|\nabla \tilde{a} \tilde{u}_{0}\right\|+\|\nabla \tilde{a} \tilde{u}\|\right) \\
\leqslant \frac{C h^{2}}{\varepsilon}\left(\left\|f_{1}\right\|_{H^{1}}+\frac{1}{\varepsilon}\left\|f_{0}\right\|+\frac{1}{\sqrt{\varepsilon}}\|f\|_{L_{\infty}}\right) .
\end{gathered}
$$

Thus,

$$
\left\|\tilde{u}-P_{h}^{\varepsilon} \tilde{u}\right\| \leqslant \frac{C h^{2}}{\varepsilon^{2}}\left(\left\|f_{0}\right\|+\varepsilon\left\|f_{1}\right\|_{H^{\prime}}\right)+\frac{C h^{2}}{\varepsilon^{3 / 2}}\|f\|_{L_{\infty}}
$$

and the desired estimate follows.

Remark A.2. Corresponding to (A.2) one may establish in case $a \in\left[H^{1}, L_{2}\right]_{1 / 2, \infty}$ $\cap L_{\infty}$ that

$$
\|u\|_{1 / 2, \infty}+\|u\|_{L_{\infty}} \leqslant C\left(\|f\|_{1 / 2, \infty}+\|u\|_{L_{\infty}}\right)
$$

uniformly in $\varepsilon$. 
The techniques of Theorem A.2 can also be used to derive the result that

$$
\left\|u-\frac{f}{a}\right\|_{L_{2}} \leqslant C \sqrt{\varepsilon}
$$

for $a, f \in H^{1 / 2, \infty} \cap L_{\infty}$, cf. Lions [14, p. 128, (5.15)] where the same estimate was proven for $a$ and $\partial \mathcal{R}$ smooth and $f \in H_{06}^{1}{ }^{2}$.

Department of Mathematics and

Center for Applied Mathematics

Cornell University

Ithaca, New York 14853

1. J. BARANGER, "On the thickness of the boundary layer in elliptic-elliptic singular perturbation problems," Numerical Analysis of Singular Perturbation Problems (P. W. Hemker and J. J. H. Miller, eds.), Academic Press, London, New York, San Francisco, 1979, pp. 395-400.

2. J. BerGH \& J. LÖFSTRÖM, Interpolation Spaces: An Introduction, Springer-Verlag, Berlin, Heidelberg, New York, 1976.

3. L. Bers, F. John \& M. SCHEChter, Partial Differential Equations, Interscience, New York, London, Sydney, 1964.

4. J. G. BESJES, "Singular perturbation problems for linear elliptic differential operators of arbitrary order. I. Degeneration to elliptic operators," J. Math. Anal. Appl., v. 49, 1975, pp. 24-46.

5. H. BRÉzis, Opérateurs Maximaux Monotones et Semi-Groupes de Contractions dans les Espaces de Hilhert, North-Holland, Amsterdam, 1973.

6. Ph. Ciarlet, The Finite Element Method for Elliptic Problems, North-Holland, Amsterdam, 1978.

7. J. Descloux, “On finite element matrices," SIAM J. Numer. Anal., v. 9, 1972, pp. 260-265.

8. J. Douglas, JR., T. Dupont \& L. B. Wahlbin, "The stability in $L^{q}$ of the $L^{2}$-projection into finite element function spaces,” Numer. Math., v. 23, 1975, pp. 193-197.

9. W. Eckhaus, Matched Asymptotic Expansions and Singular Perturbations, North-Holland, Amsterdam, 1973.

10. W. Eckhaus, Asymptotic Analysis of Singular Perturbations, North-Holland, Amsterdam, 1979.

11. P. Grisvard, Boundary Value Problems in Nonsmooth Domains, Lecture Notes 19, Department of Mathematics, University of Maryland, College Park, Maryland, 1980.

12. P. W. Hemker, A Numerical Study of Stiff Two-Point Boundary Problems, Math. Centre Tracts 80, Amsterdam, 1977.

13. A. M. ILIN, "A difference scheme for a differential equation with a small parameter multiplying the highest derivative," Mat. Zametki, v. 6, 1969, pp. 237-248; English transl. in Math. Notes, v. 6, 1969, pp. 596-602.

14. J. L. Lions, Perturbations Singulières dans les Problèmes aux Limites et en Contrôle Optimal, Lecture Notes in Math., vol. 323, Springer-Verlag, Berlin, Heidelberg, New York, 1973.

15. J. L. Lions, Quelques Méthodes de Résolution des Problèmes aux Limites Non Linéaires, Dunod, Paris, 1969.

16. J. L. Lions \& E. Magenes, Non-Homogeneous Boundary Value Problems and Applications. I, Springer-Verlag, New York, Heidelberg, Berlin, 1972.

17. J. J. H. Miller, "On the convergence, uniformly in $\varepsilon$, of difference schemes for a two point boundary singular perturbation problem," Numerical Analysis of Singular Perturbation Problems (P. W. Hemker and J. J. H. Miller, eds.), Academic Press, London, New York, San Francisco, 1979, pp. 467-474.

18. W. L. Miranker, The Computational Theory of Stiff Differential Equations, Istituto per le Applicazioni del Calcolo "Mauro Picone", Rome, 1975.

19. K. NiIJima, "On a three-point difference scheme for a singular perturbation problem without a first derivative term. I," Mem. Num. Math., v. 7, 1980.

20. K. NiIJima, "On a three-point difference scheme for a singular perturbation problem without a first derivative term. II". Mem. Num. Math., v. 7, 1980.

21. J. Nitsche \& A. H. SChatz, "On local approximation properties of $L_{2}$ projection on spline subspaces," Applicable Anal., v. 2, 1972, pp. 161-168.

22. H.-J. ReINHARDT, "A-posteriori error estimates and adaptive finite element computations for singularly perturbed one space dimensional parabolic problems," Analytical and Numerical Approaches to Asymptotic Problems in Analyis (O. Axelsson, L. S. Frank and A. Van der Sluis, eds.), North-Holland, Amsterdam, 1981, pp. 213-233. 
23. A. H. Schatz, V. Thomé \& L. B. Wahlbin, "Maximum norm stability and error estimates in parabolic finite element equations," Comm. Pure Appl. Math., v. 33, 1980, pp. 265-304.

24. A. H. Schatz \& L. B. Wahlbin, "Interior maximum norm estimates for finite element methods," Math. Comp., v. 31, 1977, pp. 414-442.

25. A. H. Schatz \& L. B. Wahlbin, "On the quasi-optimality in $L_{\infty}$ of the $\stackrel{H}{ }^{1}$ projection into finite element spaces," Math. Comp., v. 38, 1982, pp. 1-22.

26. G. I. Shiskin \& V. A. Tirov, "A difference scheme for a differential equation with two small parameters affecting the derivatives," Numer. Meth. Mechs. Cont. Media, v. 7, 1976, pp. 145-155. (Russian)

27. L. B. WAhlBin, "A quasioptimal estimate in piecewise polynomial Galerkin approximation of parabolic problems," Numerical Analysis (G. A. Watson, ed.), Lecture Notes in Math., vol. 912, Springer-Verlag, New York, Heidelberg, Berlin, 1982, pp. 230-245.

28. W. L. Wendland, Elliptic Systems in the Plane, Pitman, San Francisco, 1979.

29. M. Zlamal, "Curved elements in the finite element method. II," SIAM J. Numer. Anal., v. 11, 1974 , pp. 347-362. 